\title{
Geothermal Diatoms: Seasonal Variability in the El Tatio Geothermal Field (Altiplano, Chile)
}

\author{
Alejandro Angel1*, Irma Vila1, Carolina Díaz ${ }^{1}$, Ximena Molina1, Paola Sepúlveda² \\ ${ }^{1}$ Faculty of Science, Universidad de Chile, Santiago, Chile \\ ${ }^{2}$ Laboratorio Ambiental Centro de Ecología Aplicada, Santiago, Chile \\ Email: ^alejandro.angel@ug.uchile.cl, limnolog@uchile.cl, cardiaz@uchile.cl,mxmolinap@gmail.cl,paula.munoz@uchile.cl
}

How to cite this paper: Angel, A., Vila, I., Díaz, C., Molina, X. and Sepúlveda, P. (2018) Geothermal Diatoms: Seasonal Variability in the El Tatio Geothermal Field (Altiplano, Chile). Advances in Microbiology, 8, 211-234.

https://doi.org/10.4236/aim.2018.83015

Received: January 20, 2018

Accepted: March 26, 2018

Published: March 30, 2018

Copyright $\odot 2018$ by authors and Scientific Research Publishing Inc. This work is licensed under the Creative Commons Attribution International License (CC BY 4.0).

http://creativecommons.org/licenses/by/4.0/

\begin{abstract}
Diatom floras were examined in a high-altitude geothermal field, 4200 - 4500 $\mathrm{m}\left(29^{\circ} 19^{\prime} \mathrm{S} 68^{\circ} \mathrm{W}^{\prime}\right)$, located in the Central Andean dry Puna ecoregion or southern Altiplano. These locations include hostile environments subjecting living organisms to extreme conditions. The aim of the present study was to investigate the spatial patterns and describe the response of diatom assemblages to differences in physical and chemical variables. Different shallow $(<10 \mathrm{~cm})$ aquatic systems with variable chemical and physical conditions (fumaroles, freshwater-saline rivers and swamps) were studied seasonally during 2011-2012. The conductivity exhibited high variability ( 360 to $18340 \mu \mathrm{S} \mathrm{cm}^{-1}$ ) among the systems studied, temperature was lower in rivers and swamps $\left(6.8^{\circ} \mathrm{C}-10^{\circ} \mathrm{C}\right)$ and high in fumaroles stations $\left(30^{\circ} \mathrm{C}-37.5^{\circ} \mathrm{C}\right)$, and $\mathrm{pH}$ was lower in fumaroles than freshwater systems (3.25 to 8.97). Statistical analyses suggest that the diatoms cluster into three major groups. The most common taxa include: Achnanthidium exiguum (Grunow) Czarnecki, Cocconeis placentula var. lineata (Ehrenberg) Van Heurck, Eolimna minima (Grunow) Lange-Bertalot, Staurosirella pinnata (Ehrenberg) Williams and Round, Navicula gregaria Donkin, Nitzschia inconspicua Grunow, Nitzschia palea (Kützing) Smith, Nitzschia perminuta (Grunow) Peragallo, and Planothidium lanceolatum (Brébisson ex Kützing) Lange-Bertalot. As expected, the 20 to $200 \mu \mathrm{m}$-size fraction contained the highest numbers of diatom taxa (53 species), although an unexpectedly high number (47 species) were also found in the smaller 5 to $20 \mu \mathrm{m}$-size fraction, more associated to fumaroles and saline systems. The 180 to $2000 \mu \mathrm{m}$ size fraction contained only two species, including rosette-forming diatom Ulnaria ulna (Nitzsch) Compère, and the unicellular species Surirella chilensis Janisch, both species exclusively reported in freshwater systems. Canonical correspondence analysis (CCA) and Monte Carlo permutation tests showed clear correlations between species, conductivity, TP (total phosphorous), $\mathrm{NO}_{3}^{-}, \mathrm{HCO}_{3}^{-}, \mathrm{Mg}^{2+}$, temperature and dissolved
\end{abstract}


oxygen (DO). The gradient of ionic composition values explaining most variation in diatom assemblages ranged from waters dominated by $\mathrm{Ca}^{2+}$ and $\mathrm{SO}_{4}^{2-}$ to waters with higher proportions of $\mathrm{Na}^{+}, \mathrm{K}^{+}, \mathrm{F}^{-}, \mathrm{Li}^{+}, \mathrm{Mg}^{2+}$ and $\mathrm{Cl}^{-}$. Other factors include substrate type, presence of macrophytes, current velocity and other local environmental conditions. The results presented here enhance our understanding of diatom richness/composition in hostile environments from a high-altitude arid and semi-arid geothermal region.

\section{Keywords}

Canonical Correspondence Analysis, Cluster, Conductivity, Fumaroles, Size Fraction

\section{Introduction}

El Tatio geothermal field, one of the least known major geothermal systems in the southern hemisphere, comprise an area that contains a high heterogeneity of aquatic micro-habitats with unique physiographic conditions, high altitude $4200-4600 \mathrm{~m}$, low latitude $22^{\prime} 8^{\circ} \mathrm{S}$, high surface solar radiation $\left(280 \mathrm{~W} / \mathrm{m}^{2}\right)$, lower water boiling point $\left(86^{\circ} \mathrm{C}\right)$, and the influence of high contents of potentially toxic elements in the water [1]. These features make these systems particularly interesting in order to interpret the factors that could influence the diatom richness/composition, and the origin of diagenetic evolution of hot-spring silica deposits [2]. El Tatio is the largest known geyser field in the southern hemisphere, and the third larger field in the world after Yellowstone, USA, and Dolina Geizerov, Russia. The geothermal systems are concentrated in three main zones encompassing an area of $\sim 10 \mathrm{~km}^{2}$. Other thermal manifestations, such as small hot springs, fumaroles, and steaming soils, extend over an area of some $30 \mathrm{~km}^{2}$ at elevations from 4200 to 4600 meters [2].

The surface water discharges at a maximum temperature of $86^{\circ} \mathrm{C}$, which is the boiling point for an altitude of $4200 \mathrm{~m}$ [3]. Deep dilution of a predominant, primary high chloride $(5500 \mathrm{mg} / \mathrm{l})$ supply water derived from precipitation some $15 \mathrm{~km}$ east of El Tatio and local groundwater produces a secondary chloride water type $(4750 \mathrm{mg} / \mathrm{l})$, feeding springs over a limited area [4]. Absorption of this separated steam and carbon dioxide into local ground water and mixing with chloride waters at shallow levels produce a series of intermediate temperature $\left(40^{\circ} \mathrm{C}-70^{\circ} \mathrm{C}\right)$, low chloride, and high bicarbonate waters [2]. These conditions in chemistry water types from different geothermal microhabitats represent an interest model to be evaluated in relation to diatom richness/composition, dispersal limitation and metapopulation theories.

Several investigations of diatom floras have been carried out at individual hot springs type, or for multiple springs within a single geological setting [5] [6]. Studies of warm-spring diatoms have also been published for New Zealand [7] 
and the East African rifts [8] [9] [10]. Other diatom floras were described in hot-spring systems of Iceland, New Zealand, and Kenya [6]. Some of the common taxa described in these systems were Achnanthidium exiguum var. heterovalvum (Krasske) Czarnecki, Anomoeoneis sphaerophora Pfitzer, Brachysira brebissonii f. thermalis (Grunow) R. Ross, Diadesmis confervacea Kützing, Epithemia argus (Ehrenberg) Kützing, Nitzschia amphibia Grunow, Nitzschia inconspicua, Staurosira construens var. venter (Ehrenberg) P. B. Hamilton and Staurosirella pinnata. The historical diatom register from the Yellowstone National Park, described an extensive diatom beds of recent origin covering many square miles in the vicinity of the geyser and hot spring basins [5]. However, to compare and estimate distribution patterns, there are few available studies of diatom floras from geothermal systems in South America [11] [12], and there is no information of diatom richness/composition from the El Tatio geothermal field, considering that contain a high heterogeneity of microhabitat with unique environmental conditions.

Affinities of some freshwater diatoms with certain ions can be found in widely known richness composition [13]. For instance, a number of taxa have been characterized as preferring calcium rich or calcium poor waters [14]. It is difficult, however, to compile this information for water quality monitoring purposes in the Altiplano because the local floras studies have been scattered. It is highly probable that optima and tolerance values estimated for conductivity and major ions for common taxa among Europe, Africa and the Dry Puna ecoregion (Altiplano) would be very different, especially for aspects such as salinity, geothermal activity and mineral water dissolution.

In this study the main objectives were to: 1) describe the diatom assemblages associated with each system and to examine their spatial variability; 2) examine the degree of floral similarity/dissimilarity and size classes distribution among the systems studied; 3) use the resulting data to relate species to environmental variables; and 4) use the models to characterize the optima and tolerance values for the most common diatom taxa. Given that environmental heterogeneity can demonstrate varying and significant impacts on diatom floras, this research project emphasizes to study not only species richness/composition, but how local communities vary across seasonal changes, including chemical and physical conditions.

\section{Materials and Methods}

\subsection{Study Area}

El Tatio is a geothermal field located in northern Chile $\left(29^{\circ} 19^{\prime} \mathrm{S} 68^{\circ} \mathrm{W}^{\prime}, 4200\right.$ $4500 \mathrm{~m}$ ) at the Central Andean dry Puna ecoregion (Figure 1), a montane grassland with three main types of thermal springs [1]: springs discharging high chloride waters $(8000 \mathrm{mg} / \mathrm{l})$ located along a SW-NE trending line in the northern part of the main thermal area, more dilute, intermediate chloride pools (5000 $\mathrm{mg} / \mathrm{l}$ ) in the south-western part, and low chloride, high sulfate pools along the 


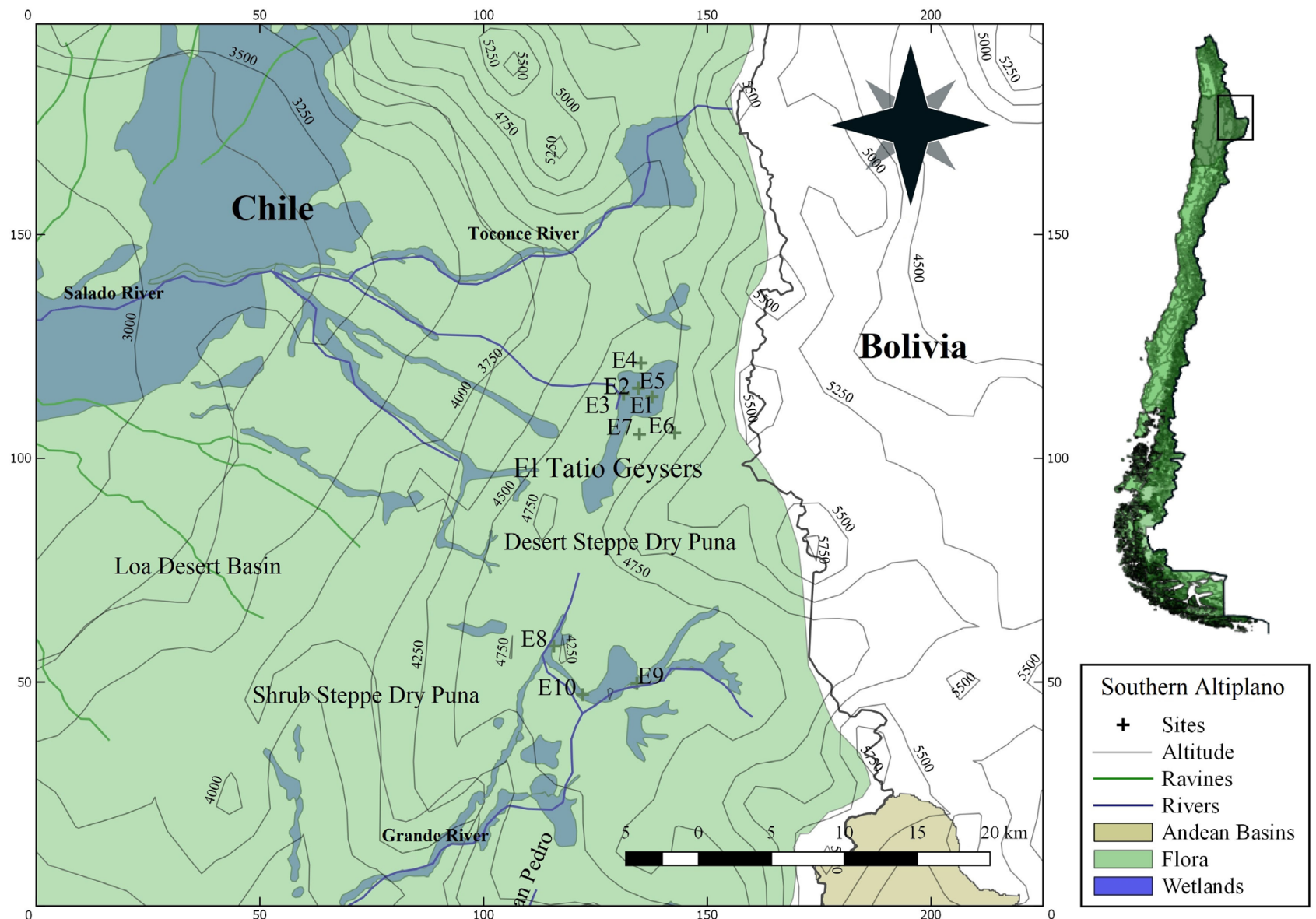

Figure 1. Location of the study sites in El Tatio Geothermal field (Altiplano, Chile).

eastern margins [1] [3] [4]. The Dry Puna ecoregion is oligothermic (mean annual temperature range: $8^{\circ} \mathrm{C}$ to $11^{\circ} \mathrm{C}$ ). Daily temperature variation reaches $35^{\circ} \mathrm{C}$ in summer and fall to $30^{\circ} \mathrm{C}$ in winter, lower night temperature fall to $5^{\circ} \mathrm{C}$ in summer and $-10^{\circ} \mathrm{C}$ in winter [1] [2] [3]. Only flora and fauna highly adapted to the extreme daily temperature variability and high-altitude live in this ecoregion.

The negative water balance is characterized by low precipitation (100 $\mathrm{mm}$ /year), very seasonal rainfall between December to March and an eight month-long dry season [3]. In this context, a variety of five aquatic systems associated to the hot springs, saline fumaroles, freshwater fumaroles, saline rivers, freshwater rivers and swamps, were sampled within two sub-basin areas (Table 1). The study sites included the Loa River basin (north area) and Salado River sub-basin (south area). The stations studied are located in two areas approximately 30 kilometers apart and were selected based on proximity and important environmental dissimilarities (Figure 2).

\subsection{Water Chemistry and Environmental Variables}

Water temperature, $\mathrm{pH}$, and conductivity were measured with portable instruments, calibrated in the field. For our study we collected 40 samples during four seasons from April, 2011 to January, 2012. The samples were collected at 10 
Table 1. Stations coordinates and altitude.

\begin{tabular}{ccccc}
\hline Sample & Location & \multicolumn{2}{c}{ Coordinates UTM-WGS (N-E) } & Altitude (m) \\
\hline E1 & Fumarole-warm spring (saline system) & 7529058 & 600390 & 4248 \\
E2 & Fumarole-warm spring (saline system) & 7529058 & 600386 & 4248 \\
E3 & Fumarole-war spring (saline system) & 7529023 & 600385 & 4250 \\
E4 & La Concha River (saline system) & 7529475 & 601391 & 4245 \\
E5 & Fumarole-warm spring (freshwater system) & 7526506 & 603998 & 4480 \\
E6 & Loa River (freshwater system) & 7529478 & 601390 & 4245 \\
E7 & Loa River (freshwater system) & 7526314 & 601583 & 4350 \\
E8 & Juana Swamp-Tocorpuri (swamps) & 7511566 & 596292 & 4202 \\
E9 & Putana Swamp high water level (swamps) & 7509247 & 602100 & 4270 \\
E10 & Putana Swamp low water level (swamps) & 7508319 & 598407 & 4271 \\
\hline
\end{tabular}

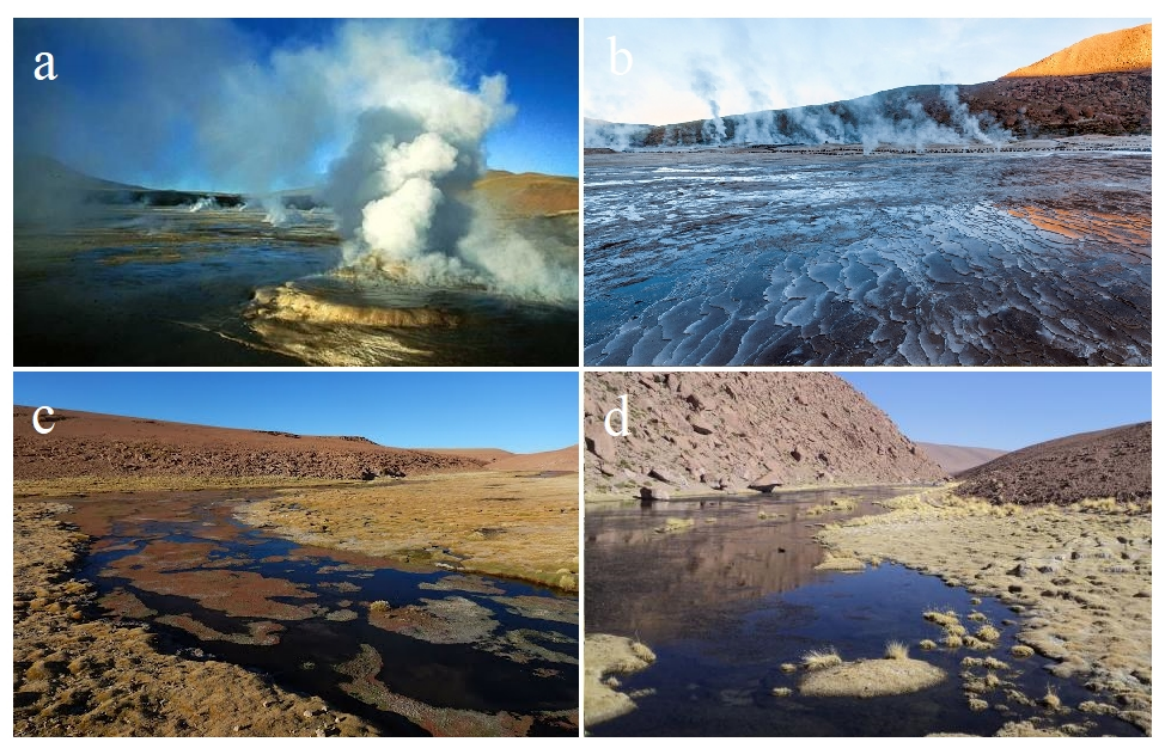

Figure 2. Habitat description. Fumaroles and active geysers ejecting water and steam through a vent (a), laminated structures, saline rivers and fumaroles associated to the geothermal field (b), Tocorpuri swamps and common Juncacae peat bogs (c), Putana swamps and common arid mountains (d).

designated sampling stations along transects selected. The analyses for individual cations $\mathrm{Li}^{+}, \mathrm{Ca}^{2+}, \mathrm{K}^{+}, \mathrm{Na}^{+}$, and $\mathrm{Mg}^{2+}$ were performed by ionic chromatography [15]. The analysis for $\mathrm{Cl}^{-}$was performed by the argentometric method, $\mathrm{SO}_{4}^{2-}$ by the gravimetric method and residual drying [15], and $\mathrm{F}^{-}$by a specific electrode with Coupled Plasma Induction/Mass spectrophotometry (ICP/MS). The analyses for nutrients, carbonates, and bicarbonate were performed by standardized APHA 2005 methods [16].

\subsection{Collection of Algal Communities}

At each site, algal communities were obtained in submerged open and unshaded areas by scraping muddy substrates or rocky riffle from epipelic sections within 
a $10 \mathrm{~m}$ study reach. Algal samples were taken from 6 randomly selected goldenbrown patches that were scraped for diatoms or collected by syringe suction into dark bottles and immediately preserved with formalin (10\% final concentration). Then, two golden-brown patches from different riffles or muddy substrates were subsequently pooled into a single sample (i.e. $\mathrm{n}=3$ ) to minimize the anticipated within-reach variation of algal assemblages, because the emphasis in the study was to examine broad (between-site) spatial patterns in benthic algal communities. Where necessary, diatom smear slides were made after carbonates were dissolved with $\mathrm{HCl}$ and organics were removed with $\mathrm{H}_{2} \mathrm{O}_{2}$. Cleaned diatom samples were mounted in Naphrax, before examination with a light microscope 1000x. Identifications were based in morphological characters according to [8] [11] [13] [17] [18] [19]. Three hundred diatoms were counted per smear slide, except where diatoms were rare, in which case all diatoms were counted.

\subsection{Statistical Analyses}

Multivariate techniques were used to examine the environmental factors associated with diatom and their distributions. Detrended correspondence analysis (DCA) suggested that a unimodal model was suitable and the data were then explored using Canonical correspondence analysis (CCA) using CANOCO 4.5 [20]. All diatoms were included in these analyses. A subset of 39 species (conditional to $0.01 \%$ to $0.33 \%$ of the total variance) was selected to simplify diagrams. Inference models were developed for $\mathrm{pH}$, Dissolved oxygen, temperature, and major ions, using the data analysis program (C2, version 1.4) and techniques according to [6]. Floral communities and sampling sites were distinguished based on percentage diatom data (forming $5 \%$ of the flora in at least one sample), using Agglomerative Hierarchical Cluster Analyses (AHC) and Heat Maps (removing all species with interquartile range lower than 0.25) carried out with XLSTAT Version 2016 (Addinsoft).

\section{Results}

\subsection{Environmental Characteristics}

The soil structure in fumaroles stations was irregular and variable with different mineral formations (Figure 2(a)), presence of laminated structures (Figure 2(b)), and salt crust layers without macrophytes. Hydrological conditions were variables among the systems studied, high conductivity and low sulfates concentration were reported in saline rivers, La Concha River station (E4), contrasting with low conductivity and high sulfates levels in the Loa River stations (E6 and E7). To the south area, is common the presence of peatland bogs (e.g. Bofedales) located in the Atacama sub-basin (stations E8, E9 and E10, Figure 2(c) \& Figure $2(d))$ and aquatic systems with very low current velocity, these condition determined low and middle values in conductivity and high levels in nutrient concentration. The largest step in the samples dendrogram occurs at 0.76 level of dissimilarity and produced five clusters, corresponding with the five type of sys- 
tems studied (Figure 3(a)).

The rivers sampled were characterized by two different types saline and freshwater systems, conductivity varied from 10 to $18,340 \mu \mathrm{Scm}^{-1}$. Median and interquartile range values for conductivity and concentration of individual ions indicated that the Loa River stations had moderate levels of salt content and were classified as sulfate calcium bicarbonate type (Figure 3(b)). However, La Concha River station showed low seasonal variability and high conductivity (range from 16,680 to $18,340 \mu \mathrm{S} \mathrm{cm}^{-1}$ ), this station was classified as sodium chloride type (Figure 3(b)). The conductivity in saline fumaroles varied from 4860 to $12,150 \mu \mathrm{S} \mathrm{cm}^{-1}$, corresponding to waters highly variable in ionic composition, with the exception of the freshwater fumarole station (E5) where conductivity showed the lowest values (range from 440 to $810 \mu \mathrm{S} \mathrm{cm}^{-1}$ ). Conductivity in swamps varied from 540 to $8020 \mu \mathrm{S} \mathrm{cm}^{-1}$, with moderate levels of electrolytes and high concentrations of $\mathrm{Ca}^{2+}, \mathrm{HCO}_{3}^{-}$and $\mathrm{SO}_{4}^{2-}$. The total concentration of $\mathrm{Ca}^{2+}$ and $\mathrm{Na}^{+}$was, however, maximum in waters with the highest proportion of $\mathrm{Cl}^{-}$among anions, mostly in saline fumaroles and saline rivers. The highest concentrations of $\mathrm{Li}^{+}, \mathrm{K}^{+}, \mathrm{F}^{-}$and $\mathrm{Cl}^{-}$were also observed in these sites (Figure 4). For instance, the total concentration of $\mathrm{Mg}^{2+}$ was maximum in waters with the highest proportion of $\mathrm{SO}_{4}^{2-}$ among anions; this condition was observed in the Loa River and Swamps stations (Figure 5). Table 2 details chemical and physical characteristics of the stations studied.

\subsection{Diatom Community Composition}

A total of 102 species and 41 genera were identified (Table 3), diatom preservation was generally good or excellent with little corrosion and minimal fragmentation, however, teratological forms were common in fumaroles stations. The Shannon-Wiener diversity index for the diatom communities ranged from 1.02
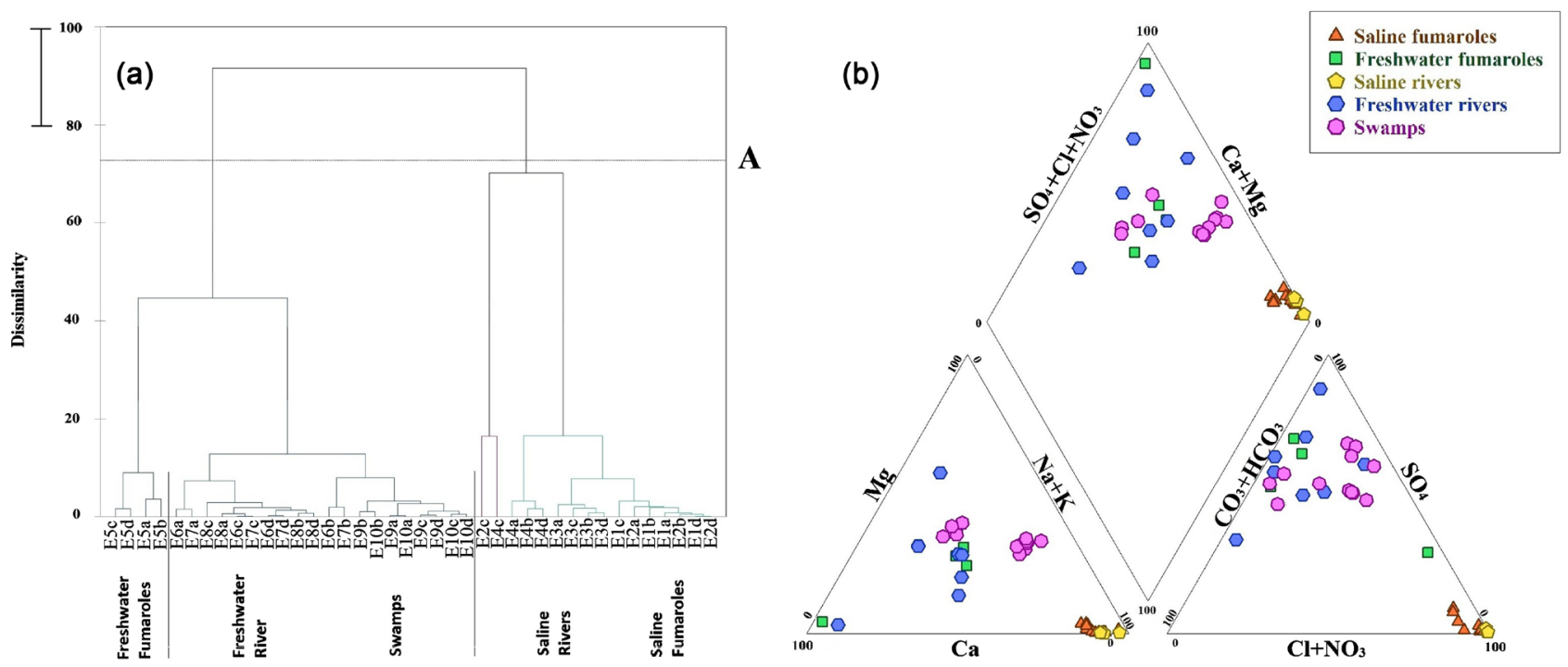

Figure 3. Herarchical analysis (a) using environmental variables (cutoff line "A" occurs at 0.76 dissimilarity and produces five clusters) and ternary diagram (b) showing major ion composition. 


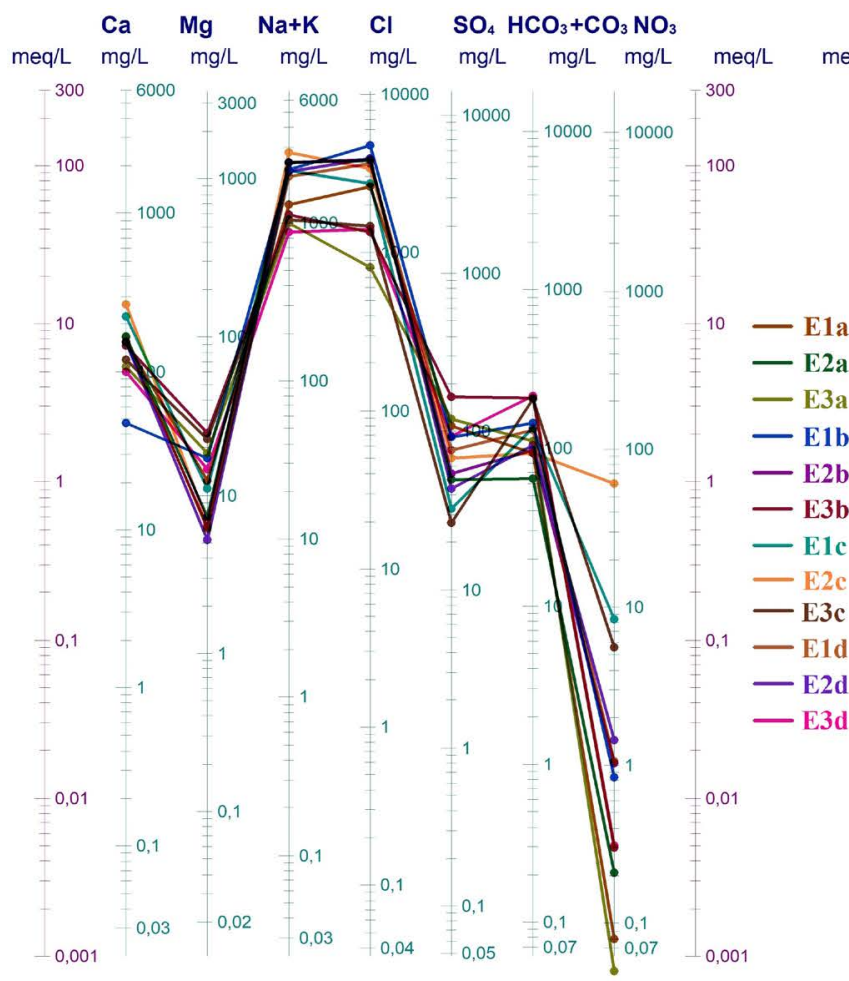

Saline Fumaroles

\section{$\mathrm{Ca} \quad \mathrm{Mg} \quad \mathrm{Na}+\mathrm{K} \quad \mathrm{Cl} \quad \mathrm{SO}_{4} \mathrm{HCO}_{3}+\mathrm{CO}_{3} \mathrm{NO}_{3}$}

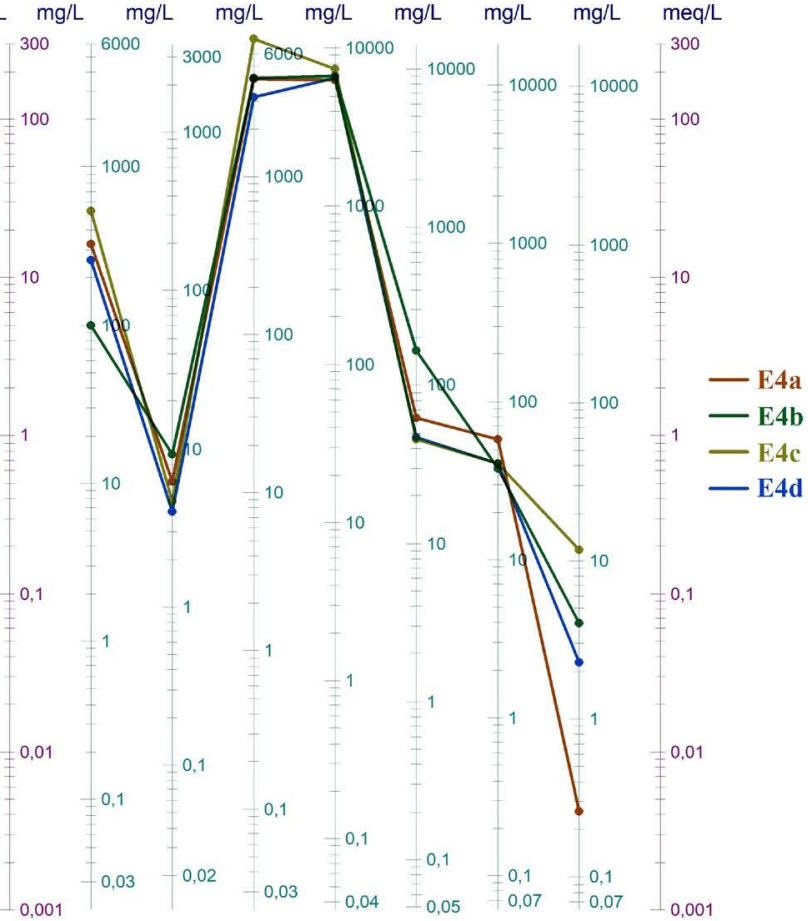

Saline Rivers

Figure 4. Vertical diagrams (Schoeller-Berkaloff) of surface water in the El tatio geothermal field. Data from this work (2010-2011) showing seasonal ion concentration (a: autumn, b: winter, c: spring, d: summer) and saline water types.

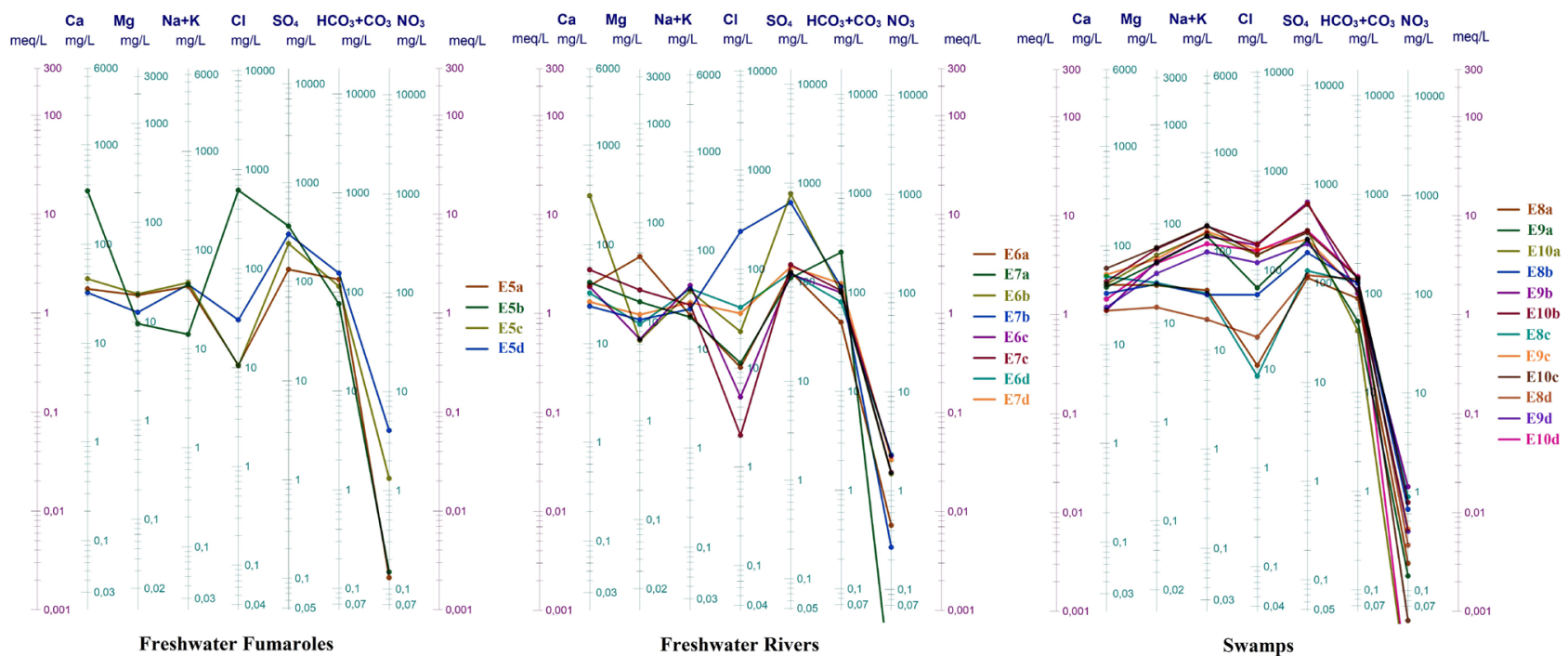

Figure 5. Vertical diagrams (Schoeller-Berkaloff) of surface water in the El tatio geothermal field. Data from this work (2010-2011) showing seasonal ion concentration (a: autumn, b: winter, c: spring, d: summer) and freshwater types.

to 2.9 with the lowest diversity at proximal hot spring locations. The most common species, forming $5 \%$ of the flora in at least one sample, for all sites were described in Figure 6. Cluster analyses using the whole dataset produced similar species patterns. The samples dendrogram produces five clusters, meanwhile, species dendrogram produces three clusters. Placing a cutoff at this point tends 
to be supported by a levelling off of the subsequent fusion points in the histogram of dissimilarity vs. agglomeration stage (Figure 6). This also has the advantage of distinguishing clusters and their associations, which is similar in CCA plot discussed below, and the floras of which have contrasting ecological requirements. Some of the most common species photographs are described in Figure 7 and Figure 8. Using the latter approach, three groups are recognized.

Group I was only formed by three species that included: Achnanthidium exiguum (Figure 7(a)), Nitzschia perminuta (Figure 8(s)), and Staurosirella leptostauron (Ehrenberg) Williams and Round. These species were most common in slightly alkaline ( $\mathrm{pH} 7.5$ - 8) low-conductivity fumaroles, freshwater rivers and margins of warm $\left(30^{\circ} \mathrm{C}-35^{\circ} \mathrm{C}\right)$ springs.

Group II was more varied and included: Halamphora acutiuscula (Kützing) Levkov, Pseudostaurosira brevistriata (Grunow) Williams and Round, Nitzschia valdecostata Lange-Bertalot and Simonsen, Navicula gregaria (Figure $7(\mathrm{j})$ ), Planothidium delicatulum (Kützing) Round and Bukhtiyarova (Figure 8(t)),

Table 2. Physical and chemical variables of freshwater and saline stations studied in the El Tatio geothermal field.

\begin{tabular}{|c|c|c|c|c|c|}
\hline Variables & $\begin{array}{c}\text { Saline } \\
\text { Fumaroles }\end{array}$ & $\begin{array}{c}\text { Freshwater } \\
\text { Fumaroles }\end{array}$ & Saline Rivers & Freshwater River & Swamps \\
\hline Conductivity & $9.3 \pm 3.1$ & $0.6 \pm 0.2$ & $17.6 \pm 0.7$ & $0.4 \pm 0.1$ & $2.3 \pm 2.1$ \\
\hline $\mathrm{pH}$ & $7.5 \pm 0.3$ & $3.5 \pm 0.2$ & $7.3 \pm 0.4$ & $8.1 \pm 0.7$ & $7.3 \pm 0.6$ \\
\hline $\mathrm{T}$ & $30.7 \pm 4.4$ & $34.8 \pm 2.3$ & $14.8 \pm 8.4$ & $9.9 \pm 0.7$ & $9.5 \pm 3.5$ \\
\hline DO & $5.2 \pm 0.6$ & $4.3 \pm 0.5$ & $7.1 \pm 0.9$ & $8.8 \pm 0.5$ & $7.6 \pm 2.4$ \\
\hline $\mathrm{Ca}^{2+}$ & $149.7 \pm 56.6$ & $104.6 \pm 107.1$ & $301.1 \pm 176.6$ & $75.5 \pm 109.4$ & $37.4 \pm 11.8$ \\
\hline $\mathrm{K}^{+}$ & $155.7 \pm 39.1$ & $13.4 \pm 4.4$ & $203.4 \pm 17.1$ & $9.4 \pm 5.1$ & $14.3 \pm 6.3$ \\
\hline $\mathrm{Na}^{+}$ & $1618.6 \pm 631.8$ & $23.1 \pm 6.2$ & $4537.5 \pm 1876.6$ & $22.1 \pm 8.2$ & $95.3 \pm 54.5$ \\
\hline $\mathrm{Li}^{+}$ & $13.5 \pm 4.9$ & $0.8 \pm 0.5$ & $30.1 \pm 2.2$ & $0.2 \pm 0.2$ & $0.4 \pm 0.2$ \\
\hline $\mathrm{Mg}^{2+}$ & $12.7 \pm 6.8$ & $17.5 \pm 9.2$ & $6.5 \pm 2.3$ & $14.8 \pm 4.3$ & $37.4 \pm 13.5$ \\
\hline $\mathrm{Cl}^{-}$ & $2820.8 \pm 1290.9$ & $19.8 \pm 9.5$ & $6698.5 \pm 472.4$ & $120.1 \pm 117.1$ & $103.9 \pm 67.1$ \\
\hline $\mathrm{NO}_{2}$ & $139.4 \pm 242.2$ & $0.8 \pm 0.4$ & $237.1 \pm 202.5$ & $1.8 \pm 1.3$ & $4.1 \pm 3.4$ \\
\hline $\mathrm{NO}_{3}^{-}$ & $6.6 \pm 17.1$ & $1.62 \pm 0.9$ & $4.6 \pm 5.1$ & $1.9 \pm 1.4$ & $0.4 \pm 0.3$ \\
\hline $\mathrm{SO}_{4}^{2-}$ & $77.4 \pm 40.4$ & $260.7 \pm 99.5$ & $80.1 \pm 57.8$ & $274.6 \pm 73.2$ & $\begin{array}{c}304.1 \pm \\
176.8\end{array}$ \\
\hline $\mathrm{F}^{-}$ & $1.3 \pm 0.9$ & $0.4 \pm 0.1$ & $2.8 \pm 2.7$ & $0.2 \pm 0.1$ & $0.3 \pm 0.1$ \\
\hline TKN & $2.9 \pm 1.8$ & $10.2 \pm 7.1$ & $1.8 \pm 1.6$ & $1.9 \pm 1.1$ & $1.2 \pm 0.7$ \\
\hline $\mathrm{TP}$ & $1.1 \pm 0.8$ & $0.9 \pm 0.6$ & $2.1 \pm 2.8$ & $0.7 \pm 0.4$ & $0.5 \pm 0.4$ \\
\hline $\mathrm{PO}_{4}^{2-}$ & $0.5 \pm 0.2$ & $0.2 \pm 0.1$ & $1.4 \pm 2.1$ & $0.3 \pm 0.2$ & $0.2 \pm 0.1$ \\
\hline $\mathrm{HCO}_{3}^{-}$ & $132.5 \pm 50.3$ & $82.5 \pm 23.6$ & $44.5 \pm 9.1$ & $134.1 \pm 54.2$ & $109.9 \pm 35.1$ \\
\hline $\mathrm{CaCO}_{3}$ & $0.9 \pm 0.5$ & $0.9 \pm 0.5$ & $0.5 \pm 0.7$ & $0.9 \pm 0.5$ & $0.5 \pm 0.3$ \\
\hline
\end{tabular}

Conductivity $(\mathrm{mS} / \mathrm{cm})$, Temperature $\left({ }^{\circ} \mathrm{C}\right)$, Dissolved Oxygen (DO), Total phosphorous (TP), Total Kjeldahl Nitrogen $(\mathrm{TKN})$ and Ionic Composition values in $(\mathrm{mg} / \mathrm{L})$. 
Table 3. Diatom species, occurrence and ecology in aquatic systems from the El Tatio geothermal field. Occurrence: number of presence in stations during all the seasons. The algae were classified as: abundant $\left(>10^{3} \mathrm{cells} / \mathrm{mm}^{3}\right)$; common $\left(10^{3}-10^{2} \mathrm{cells} / \mathrm{mm}^{3}\right)$, occasional $\left(10^{2}-10\right.$ cells $\left./ \mathrm{mm}^{3}\right)$ or rare $\left(<10\right.$ cells $\left./ \mathrm{mm}^{3}\right)$.

\begin{tabular}{|c|c|c|c|c|c|c|c|c|}
\hline Taxa & Code & $\begin{array}{c}\text { Saline } \\
\text { Fumaroles }\end{array}$ & $\begin{array}{l}\text { Saline } \\
\text { Rivers }\end{array}$ & $\begin{array}{l}\text { Freshwater } \\
\text { Fumaroles }\end{array}$ & $\begin{array}{c}\text { Freshwater } \\
\text { Rivers }\end{array}$ & Swamps & Occurrence & Ecology \\
\hline Achnanthes atacamae Hustedt & Ac at & $\mathrm{R}$ & $\mathrm{N}$ & $\mathrm{N}$ & $\mathrm{N}$ & $\mathrm{R}$ & 2 & $\mathrm{E}$ \\
\hline Achnanthes sp1 & Acsp & $\mathrm{N}$ & $\mathrm{N}$ & $\mathrm{N}$ & $\mathrm{N}$ & $\mathrm{R}$ & 1 & B \\
\hline Achnanthes thermalis var. rumrichorum Lange-Bertalot & Acth & $\mathrm{N}$ & $\mathrm{R}$ & $\mathrm{N}$ & $\mathrm{N}$ & $\mathrm{N}$ & 1 & B \\
\hline Achnanthidium exiguum (Grunow) Czarnecki & Ac ex & $\mathrm{C}$ & $\mathrm{O}$ & A & A & $\mathrm{R}$ & 21 & $\mathrm{E}$ \\
\hline Achnanthidium minutissimum (Kützing) Czarnecki & $A c m i$ & $\mathrm{O}$ & $\mathrm{C}$ & $\mathrm{C}$ & $\mathrm{O}$ & $\mathrm{O}$ & 3 & $\mathrm{~T}$ \\
\hline Adlafia minuscula (Grunow) Lange-Bertalot & Admi & $\mathrm{N}$ & $\mathrm{N}$ & $\mathrm{C}$ & $\mathrm{O}$ & $\mathrm{O}$ & 8 & A \\
\hline Amphora pediculus (Kützing) Grunow & Ampe & $\mathrm{N}$ & $\mathrm{N}$ & $\mathrm{N}$ & $\mathrm{N}$ & $\mathrm{O}$ & 2 & $\mathrm{~T}$ \\
\hline Amphora sp1 & $A m s p 1$ & $\mathrm{C}$ & $\mathrm{C}$ & $\mathrm{N}$ & $\mathrm{N}$ & $\mathrm{N}$ & 10 & B \\
\hline Amphora sp2 & $A m s p 2$ & $\mathrm{C}$ & $\mathrm{O}$ & $\mathrm{N}$ & $\mathrm{R}$ & $\mathrm{N}$ & 6 & B \\
\hline Amphora sp3 & $A m s p 3$ & $\mathrm{R}$ & $\mathrm{N}$ & $\mathrm{N}$ & $\mathrm{N}$ & $\mathrm{R}$ & 2 & B \\
\hline Brachysira aponina Kützing & Brap & $\mathrm{O}$ & $\mathrm{O}$ & $\mathrm{N}$ & $\mathrm{N}$ & $\mathrm{N}$ & 3 & $\mathrm{E}$ \\
\hline Caloneis bacillum (Grunow) Cleve & Ca ba & $\mathrm{N}$ & $\mathrm{N}$ & $\mathrm{O}$ & $\mathrm{R}$ & $\mathrm{R}$ & 3 & $\mathrm{~T}$ \\
\hline Caloneis sp1 & Ca $s p$ & $\mathrm{~N}$ & $\mathrm{~N}$ & $\mathrm{~N}$ & $\mathrm{~N}$ & $\mathrm{O}$ & 1 & $\mathrm{~T}$ \\
\hline Cocconeis placentula var. euglypta (Ehrenberg) Grunow & Co pl & $\mathrm{N}$ & $\mathrm{N}$ & $\mathrm{N}$ & $\mathrm{O}$ & $\mathrm{N}$ & 1 & $\mathrm{~T}$ \\
\hline Cocconeis placentula var. lineata (Ehrenberg) Van Heurck & Coli & $\mathrm{O}$ & $\mathrm{A}$ & $\mathrm{N}$ & A & A & 28 & $\mathrm{~T}$ \\
\hline Cymbellonitzschia sp1 & Cysp 1 & $\mathrm{O}$ & $\mathrm{N}$ & $\mathrm{N}$ & $\mathrm{N}$ & $\mathrm{N}$ & 1 & $\mathrm{E}$ \\
\hline Denticula elegans Kützing & De el & $\mathrm{N}$ & $\mathrm{O}$ & $\mathrm{N}$ & $\mathrm{N}$ & $\mathrm{N}$ & 1 & A \\
\hline Denticula kuetzingii Grunow & De ku & $\mathrm{O}$ & $\mathrm{N}$ & $\mathrm{N}$ & $\mathrm{N}$ & $\mathrm{N}$ & 2 & A \\
\hline Denticula subtilis Grunow & Desu & $\mathrm{A}$ & $\mathrm{O}$ & $\mathrm{N}$ & $\mathrm{N}$ & $\mathrm{N}$ & 11 & A \\
\hline Denticula thermalis Kützing & De th & $\mathrm{O}$ & $\mathrm{R}$ & $\mathrm{N}$ & $\mathrm{N}$ & $\mathrm{N}$ & 4 & A \\
\hline Denticula valida (Pedicino) Grunow & De va & $\mathrm{N}$ & $\mathrm{O}$ & $\mathrm{N}$ & $\mathrm{N}$ & $\mathrm{N}$ & 1 & A \\
\hline Diadesmis confervacea Kützing & Dico & $\mathrm{R}$ & $\mathrm{N}$ & $\mathrm{N}$ & $\mathrm{N}$ & $\mathrm{N}$ & 5 & $\mathrm{E}$ \\
\hline Encyonema chilense Krammer & Ench & $\mathrm{N}$ & $\mathrm{N}$ & $\mathrm{N}$ & $\mathrm{N}$ & $\mathrm{R}$ & 1 & $\mathrm{~N}$ \\
\hline Encyonopsis microcephala (Grunow) Krammer & En mi & $\mathrm{N}$ & $\mathrm{N}$ & $\mathrm{O}$ & $\mathrm{N}$ & $\mathrm{N}$ & 1 & A \\
\hline Eolimna minima (Grunow) Lange-Bertalot & Eo mi & $\mathrm{R}$ & $\mathrm{N}$ & $\mathrm{C}$ & $\mathrm{C}$ & $\mathrm{C}$ & 16 & $\mathrm{P}$ \\
\hline Epithemia adnata (Kützing) Brébisson & Ep ad & $\mathrm{N}$ & $\mathrm{N}$ & $\mathrm{N}$ & $\mathrm{R}$ & $\mathrm{N}$ & 1 & $\mathrm{~B}$ \\
\hline Eunotia incisa W.Smith ex W. Gregory & Eu in & $\mathrm{N}$ & $\mathrm{N}$ & $\mathrm{N}$ & $\mathrm{N}$ & $\mathrm{R}$ & 1 & A \\
\hline Fragilaria capucina Desmazières & Frca & $\mathrm{R}$ & $\mathrm{N}$ & $\mathrm{C}$ & $\mathrm{C}$ & $\mathrm{O}$ & 10 & $\mathrm{~T}$ \\
\hline $\begin{array}{l}\text { Fragilaria capucina var. vaucheriae (Kützing) } \\
\text { Lange-Bertalot }\end{array}$ & Fr va & $\mathrm{R}$ & $\mathrm{N}$ & $\mathrm{N}$ & $\mathrm{C}$ & $\mathrm{C}$ & 16 & A \\
\hline Fragilaria construens (Ehrenberg) Grunow & Frco & $\mathrm{O}$ & $\mathrm{N}$ & $\mathrm{N}$ & $\mathrm{O}$ & $\mathrm{R}$ & 1 & $\mathrm{~T}$ \\
\hline Fragilaria fasciculata (C. Agardh) Lange-Bertalot & Fr fa & $\mathrm{O}$ & $\mathrm{N}$ & $\mathrm{N}$ & $\mathrm{N}$ & $\mathrm{R}$ & 8 & $\mathrm{~T}$ \\
\hline Geissleria sp1 & Ge sp & $\mathrm{N}$ & $\mathrm{N}$ & $\mathrm{N}$ & $\mathrm{R}$ & $\mathrm{R}$ & 2 & $\mathrm{P}$ \\
\hline Gomphonema angustatum (Kützing) Rabenhorst & Go ag & $\mathrm{N}$ & $\mathrm{N}$ & $\mathrm{N}$ & $\mathrm{O}$ & $\mathrm{R}$ & 5 & B \\
\hline
\end{tabular}




\begin{tabular}{|c|c|c|c|c|c|c|c|c|}
\hline Gomphonema angustum C. Agardh & Go an & $\mathrm{N}$ & $\mathrm{N}$ & $\mathrm{O}$ & $\mathrm{N}$ & $\mathrm{N}$ & 1 & B \\
\hline Gomphonema gracile Ehrenberg & Go gr & $\mathrm{N}$ & $\mathrm{N}$ & $\mathrm{N}$ & $\mathrm{O}$ & $\mathrm{N}$ & 1 & $\mathrm{E}$ \\
\hline Gomphonema parvulum (Kützing) Kützing & Go pa & $\mathrm{N}$ & $\mathrm{N}$ & $\mathrm{N}$ & $\mathrm{O}$ & $\mathrm{R}$ & 3 & $\mathrm{E}$ \\
\hline Gomphonema pseudoaugur Lange-Bertalot & Go ps & $\mathrm{N}$ & $\mathrm{N}$ & $\mathrm{C}$ & $\mathrm{C}$ & $\mathrm{R}$ & 5 & $\mathrm{E}$ \\
\hline Gomphonema sp1 & Go sp & $\mathrm{N}$ & $\mathrm{N}$ & $\mathrm{N}$ & $\mathrm{N}$ & $\mathrm{R}$ & 1 & $\mathrm{E}$ \\
\hline Gomphonema tristigmatum Metzeltin \& Lange-Bertalot & Go tr & $\mathrm{N}$ & $\mathrm{N}$ & $\mathrm{N}$ & $\mathrm{N}$ & $\mathrm{R}$ & 1 & $\mathrm{E}$ \\
\hline Halamphora acutiuscula (Kützing) Levkov & Ha ac & $\mathrm{C}$ & $\mathrm{C}$ & $\mathrm{N}$ & $\mathrm{N}$ & $\mathrm{N}$ & 12 & A \\
\hline Halamphora atacamae (Frenguelli) Levkov (fossil) & Ha at & $\mathrm{R}$ & $\mathrm{O}$ & $\mathrm{N}$ & $\mathrm{N}$ & $\mathrm{N}$ & 3 & B \\
\hline Halamphora coffeiformis (C. Agardh) Levkov & Ha co & $\mathrm{O}$ & $\mathrm{N}$ & $\mathrm{N}$ & $\mathrm{N}$ & $\mathrm{N}$ & 3 & B \\
\hline Halamphora veneta (Kützing) Levkov & Ha ve & $\mathrm{R}$ & $\mathrm{O}$ & $\mathrm{N}$ & $\mathrm{N}$ & $\mathrm{O}$ & 6 & $\mathrm{~T}$ \\
\hline Hippodonta hungarica Lange-Bertalot & Hi hg & $\mathrm{N}$ & $\mathrm{N}$ & $\mathrm{N}$ & $\mathrm{N}$ & $\mathrm{R}$ & 1 & $\mathrm{E}$ \\
\hline Lemnicola hungarica (Grunow) Round \& Basson & Le hg & $\mathrm{N}$ & $\mathrm{N}$ & $\mathrm{N}$ & $\mathrm{N}$ & $\mathrm{O}$ & 5 & B \\
\hline Luticola mollis Lange-Bertalot \& U.Rumrich & Lu mo & $\mathrm{N}$ & $\mathrm{N}$ & $\mathrm{N}$ & $\mathrm{N}$ & $\mathrm{R}$ & 1 & A \\
\hline Mastogloia elliptica (C. Agardh) Cleve & Ma el & $\mathrm{R}$ & $\mathrm{N}$ & $\mathrm{N}$ & $\mathrm{N}$ & $\mathrm{N}$ & 3 & $\mathrm{E}$ \\
\hline Mayamaea atomus (Kützing) Lange-Bertalot & Myat & $\mathrm{N}$ & $\mathrm{N}$ & $\mathrm{C}$ & $\mathrm{O}$ & $\mathrm{O}$ & 7 & $\mathrm{E}$ \\
\hline Navicula cincta (Ehrenberg) Ralfs & $\mathrm{Naci}$ & $\mathrm{O}$ & $\mathrm{N}$ & $\mathrm{O}$ & $\mathrm{C}$ & $\mathrm{C}$ & 11 & A \\
\hline Navicula cryptocephala Kützing & $\mathrm{NaCr}$ & $\mathrm{N}$ & $\mathrm{N}$ & $\mathrm{O}$ & $\mathrm{N}$ & $\mathrm{N}$ & 1 & $\mathrm{~T}$ \\
\hline Navicula cryptotenella Lange-Bertalot & Nact & $\mathrm{O}$ & $\mathrm{O}$ & $\mathrm{N}$ & $\mathrm{N}$ & $\mathrm{N}$ & 4 & B \\
\hline Navicula gregaria Donkin & Nagr & $\mathrm{N}$ & $\mathrm{N}$ & $\mathrm{N}$ & $\mathrm{N}$ & $\mathrm{C}$ & 7 & $\mathrm{E}$ \\
\hline Navicula lauca U. Rumrich \& Lange-Bertalot & Nala & $\mathrm{O}$ & $\mathrm{R}$ & $\mathrm{N}$ & $\mathrm{O}$ & $\mathrm{O}$ & 15 & B \\
\hline Navicula microdigitoradiata Lange-Bertalot & $\mathrm{Nami}$ & $\mathrm{R}$ & $\mathrm{N}$ & $\mathrm{N}$ & $\mathrm{N}$ & $\mathrm{N}$ & 1 & B \\
\hline Navicula parinacota U. Rumrich \& Lange-Bertalot & Napa & $\mathrm{O}$ & $\mathrm{R}$ & $\mathrm{N}$ & $\mathrm{N}$ & $\mathrm{R}$ & 4 & B \\
\hline Navicula pseudogracilis Hustedt & Naps & $\mathrm{R}$ & $\mathrm{N}$ & $\mathrm{N}$ & $\mathrm{N}$ & $\mathrm{N}$ & 1 & $\mathrm{~N}$ \\
\hline Navicula salinicola Hustedt & Nasa & $\mathrm{C}$ & $\mathrm{C}$ & $\mathrm{N}$ & $\mathrm{N}$ & $\mathrm{N}$ & 13 & B \\
\hline Navicula sp1 & Na sp 1 & $\mathrm{O}$ & $\mathrm{O}$ & $\mathrm{N}$ & $\mathrm{N}$ & $\mathrm{N}$ & 3 & B \\
\hline Navicula tripunctata (O. F. Müller) Bory & Na tr & $\mathrm{N}$ & $\mathrm{N}$ & $\mathrm{N}$ & $\mathrm{N}$ & $\mathrm{C}$ & 4 & B \\
\hline Navicula veneta Kützing & Na ve & $\mathrm{O}$ & $\mathrm{O}$ & $\mathrm{N}$ & $\mathrm{N}$ & $\mathrm{N}$ & 5 & B \\
\hline Navicula viridula (Kützing) Ehrenberg & Na vi & $\mathrm{N}$ & $\mathrm{N}$ & $\mathrm{N}$ & $\mathrm{R}$ & $\mathrm{R}$ & 2 & B \\
\hline Naviculadicta chilensis (Krasske) Lange-Bertalot & NVch & $\mathrm{N}$ & $\mathrm{N}$ & $\mathrm{N}$ & $\mathrm{O}$ & $\mathrm{R}$ & 3 & B \\
\hline Navicymbula pusilla (Grunow) Krammer & $N c p u$ & $\mathrm{R}$ & $\mathrm{N}$ & $\mathrm{N}$ & $\mathrm{N}$ & $\mathrm{N}$ & 2 & A \\
\hline Nitzschia accedens var. chilensis R.M.Patrick & Nt ac & $\mathrm{R}$ & $\mathrm{N}$ & $\mathrm{N}$ & $\mathrm{N}$ & $\mathrm{N}$ & 1 & B \\
\hline Nitzschia amphibia Grunow & Nt am & $\mathrm{N}$ & $\mathrm{N}$ & $\mathrm{C}$ & $\mathrm{N}$ & $\mathrm{N}$ & 1 & A \\
\hline Nitzschia bacillum Hustedt & Nt ba & $\mathrm{O}$ & $\mathrm{O}$ & $\mathrm{C}$ & $\mathrm{O}$ & $\mathrm{N}$ & 10 & B \\
\hline Nitzschia fonticola (Grunow) Grunow & Nt fo & $\mathrm{O}$ & $\mathrm{N}$ & $\mathrm{C}$ & $\mathrm{C}$ & $\mathrm{C}$ & 9 & $\mathrm{~B}$ \\
\hline Nitzschia gracilis Hantzsch & Nt $g r$ & $\mathrm{~N}$ & $\mathrm{O}$ & $\mathrm{N}$ & $\mathrm{N}$ & $\mathrm{O}$ & 4 & $\mathrm{P}$ \\
\hline Nitzschia halloyii N. I. Maidana \& N. Herbst & Nt ha & $\mathrm{R}$ & $\mathrm{N}$ & $\mathrm{N}$ & $\mathrm{N}$ & $\mathrm{O}$ & 6 & B \\
\hline Nitzschia inconspicua Grunow & Nt in & C & $\mathrm{O}$ & $\mathrm{O}$ & $\mathrm{N}$ & A & 17 & B \\
\hline
\end{tabular}




\begin{tabular}{|c|c|c|c|c|c|c|c|c|}
\hline Nitzschia latens Hustedt & Nt la & $\mathrm{R}$ & $\mathrm{O}$ & $\mathrm{N}$ & $\mathrm{N}$ & $\mathrm{N}$ & 4 & B \\
\hline Nitzschia liebetruthii Rabenhorst & Nt li & $\mathrm{O}$ & $\mathrm{N}$ & $\mathrm{N}$ & $\mathrm{N}$ & $\mathrm{R}$ & 7 & B \\
\hline Nitzschia linearis $\mathrm{W}$. Smith & Nt $\ln$ & $\mathrm{N}$ & $\mathrm{N}$ & $\mathrm{O}$ & $\mathrm{N}$ & $\mathrm{R}$ & 2 & $\mathrm{~T}$ \\
\hline Nitzschia palea (Kützing) W. Smith & Ntpa & $\mathrm{C}$ & $\mathrm{O}$ & A & $\mathrm{C}$ & $\mathrm{C}$ & 20 & $\mathrm{~T}$ \\
\hline Nitzschia perminuta (Grunow) M. Peragallo & Ntpe & $\mathrm{O}$ & $\mathrm{N}$ & $\mathrm{O}$ & $\mathrm{C}$ & $\mathrm{C}$ & 16 & B \\
\hline Nitzschia pusilla Grunow & $N t p u$ & $\mathrm{O}$ & $\mathrm{C}$ & $\mathrm{N}$ & $\mathrm{N}$ & $\mathrm{R}$ & 7 & $\mathrm{E}$ \\
\hline Nitzschia sp1 & Nt sp & $\mathrm{O}$ & $\mathrm{A}$ & A & A & A & 26 & B \\
\hline Nitzschia subrostratoides Cholnoky & Nt su & $\mathrm{N}$ & $\mathrm{N}$ & $\mathrm{N}$ & $\mathrm{N}$ & $\mathrm{R}$ & 1 & B \\
\hline Nitzschia valdecostata Lange-Bertalot \& Simonsen & Nt va & $\mathrm{O}$ & $\mathrm{C}$ & $\mathrm{O}$ & $\mathrm{O}$ & $\mathrm{C}$ & 21 & B \\
\hline Pinnularia subrostrata (A. Cleve) Cleve-Euler & Pisu & $\mathrm{N}$ & $\mathrm{N}$ & $\mathrm{O}$ & $\mathrm{O}$ & $\mathrm{R}$ & 3 & B \\
\hline Planothidium chilense (Hustedt) Lange-Bertalot & Plch & $\mathrm{N}$ & $\mathrm{N}$ & $\mathrm{N}$ & $\mathrm{O}$ & $\mathrm{R}$ & 2 & B \\
\hline $\begin{array}{c}\text { Planothidium delicatulum (Kützing) Round \& } \\
\text { Bukhtiyarova }\end{array}$ & Pl de & $\mathrm{R}$ & $\mathrm{N}$ & $\mathrm{N}$ & $\mathrm{R}$ & $\mathrm{C}$ & 10 & $\mathrm{~T}$ \\
\hline $\begin{array}{c}\text { Planothidium frecuentissimum (Lange-Bertalot) } \\
\text { Lange-Bertalot }\end{array}$ & Pl fr & $\mathrm{N}$ & $\mathrm{N}$ & $\mathrm{O}$ & $\mathrm{C}$ & $\mathrm{O}$ & 12 & B \\
\hline $\begin{array}{c}\text { Planothidium lanceolatum (Brébisson ex Kützing) } \\
\text { Lange-Bertalot }\end{array}$ & Plla & $\mathrm{N}$ & $\mathrm{R}$ & $\mathrm{O}$ & $\mathrm{O}$ & A & 13 & $\mathrm{~T}$ \\
\hline $\begin{array}{c}\text { Planothidium renei (Lange-Bertalot \& Rol.Schmidt) Van de } \\
\text { Vijver }\end{array}$ & Plre & $\mathrm{N}$ & $\mathrm{N}$ & $\mathrm{N}$ & $\mathrm{N}$ & $\mathrm{R}$ & 5 & B \\
\hline Planothidium sp1 & $P l s p$ & $\mathrm{R}$ & $\mathrm{C}$ & $\mathrm{N}$ & $\mathrm{N}$ & $\mathrm{N}$ & 4 & B \\
\hline $\begin{array}{c}\text { Psamothidium subatomoides (Hustedt) Bukhtiyrova \& } \\
\text { Round }\end{array}$ & Ps su & $\mathrm{N}$ & $\mathrm{N}$ & $\mathrm{N}$ & $\mathrm{O}$ & $\mathrm{N}$ & 2 & B \\
\hline $\begin{array}{c}\text { Pseudostaurosira brevistriata (Grunow) D.M. Williams \& } \\
\text { Round }\end{array}$ & Ps br & $\mathrm{O}$ & $\mathrm{C}$ & $\mathrm{N}$ & $\mathrm{N}$ & $\mathrm{O}$ & 7 & $\mathrm{~T}$ \\
\hline Rhoicosphenia abbreviata (C. Agardh) Lange-Bertalot & $R h a b$ & $\mathrm{~N}$ & $\mathrm{~N}$ & $\mathrm{~N}$ & $\mathrm{~N}$ & $\mathrm{O}$ & 4 & $\mathrm{~T}$ \\
\hline Rhopalodia acuminata Krammer & $R h a c$ & $\mathrm{~N}$ & $\mathrm{~N}$ & $\mathrm{~N}$ & $\mathrm{R}$ & $\mathrm{N}$ & 1 & B \\
\hline Rhopalodia gibba (Ehrenberg) Otto Müller & Rhgi & $\mathrm{N}$ & $\mathrm{N}$ & $\mathrm{N}$ & $\mathrm{N}$ & $\mathrm{R}$ & 1 & $\mathrm{~T}$ \\
\hline Staurosira aventralis Lange-Bertalot \& Rumrich & Stav & $\mathrm{N}$ & $\mathrm{N}$ & $\mathrm{N}$ & $\mathrm{N}$ & $\mathrm{R}$ & 1 & B \\
\hline Staurosira brevistriata (Grunow) Grunow & St br & $\mathrm{C}$ & $\mathrm{O}$ & $\mathrm{N}$ & $\mathrm{N}$ & $\mathrm{O}$ & 8 & $\mathrm{~T}$ \\
\hline Staurosira subsalina (Hustedt) Lange-Bertalot & St su & $\mathrm{O}$ & $\mathrm{C}$ & $\mathrm{N}$ & $\mathrm{O}$ & $\mathrm{O}$ & 7 & $\mathrm{~T}$ \\
\hline Staurosirella leptostauron (Ehrenberg) & St le & $\mathrm{C}$ & $\mathrm{O}$ & $\mathrm{N}$ & $\mathrm{O}$ & $\mathrm{C}$ & 13 & $\mathrm{~T}$ \\
\hline Staurosirella pinnata (Ehrenberg) D. M. Williams \& Round & St pi & $\mathrm{R}$ & $\mathrm{R}$ & $\mathrm{N}$ & $\mathrm{N}$ & $\mathrm{O}$ & 10 & $\mathrm{~T}$ \\
\hline Stephanodiscus sp1 & St $s p$ & $\mathrm{~N}$ & $\mathrm{~N}$ & $\mathrm{~N}$ & $\mathrm{~N}$ & $\mathrm{R}$ & 1 & $\mathrm{P}$ \\
\hline Surirella angusta Kützing & Su an & $\mathrm{N}$ & $\mathrm{N}$ & $\mathrm{O}$ & $\mathrm{N}$ & $\mathrm{N}$ & 1 & $\mathrm{~T}$ \\
\hline Surirella chilensis $\mathrm{C}$. Janisch & Su ch & $\mathrm{R}$ & $\mathrm{N}$ & $\mathrm{N}$ & $\mathrm{N}$ & $\mathrm{N}$ & 1 & $\mathrm{E}$ \\
\hline Synedra goulardii Brébisson ex Cleve \& Grunow & Sygo & $\mathrm{N}$ & $\mathrm{N}$ & $\mathrm{N}$ & $\mathrm{N}$ & $\mathrm{R}$ & 2 & $\mathrm{E}$ \\
\hline Tryblionella hungarica (Grunow) Frenguelli & $\operatorname{Tr} h u$ & $\mathrm{~N}$ & $\mathrm{~N}$ & $\mathrm{O}$ & $\mathrm{N}$ & $\mathrm{N}$ & 3 & B \\
\hline Ulnaria ulna (Nitzsch) Compère & Ul ul & $\mathrm{N}$ & $\mathrm{N}$ & $\mathrm{C}$ & $\mathrm{C}$ & $\mathrm{R}$ & 12 & $\mathrm{E}$ \\
\hline
\end{tabular}

Aerophilic (A): Benthic (B): Epiphytic (E): Planktonic (P): Tychoplanktonic (T): Non presence (N). 


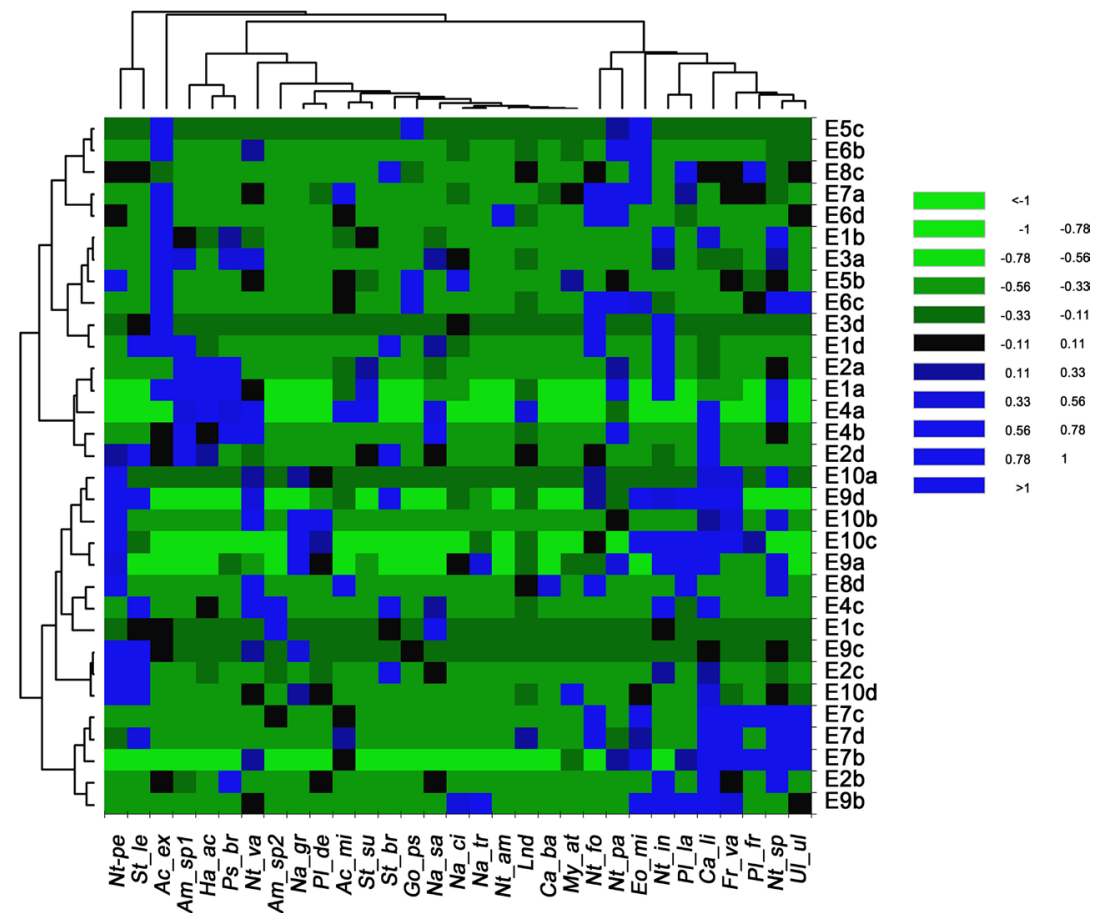

Figure 6. Heat map of diatom taxa forming $5 \%$ of the flora in at least one sample. The colors key is shown to the right (red color represent low association, green color represent high association and black color represent intermediate association), histograms are shown upper and to the left. All associations were determined by saline and freshwater conditions for both species and samples respectively (sample codes and species acronyms are described in Table 1 and Table 3).
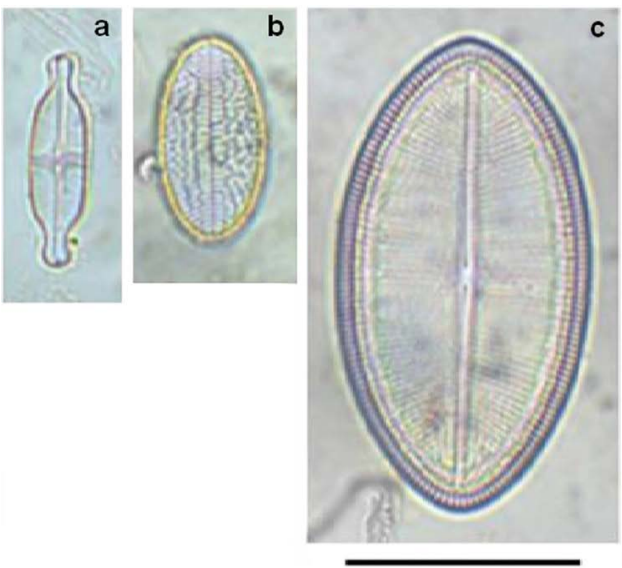

$20 \mu \mathrm{m}$
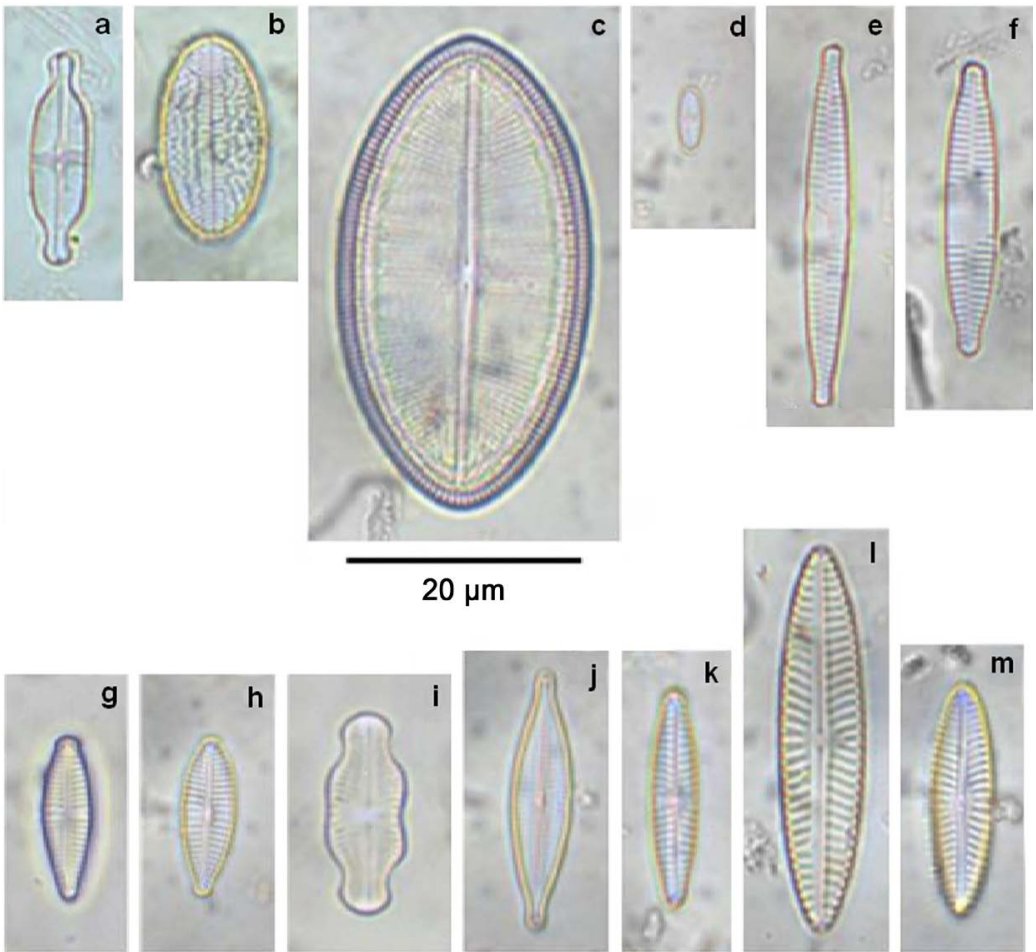

Figure 7. Light microscopy photographs of diatoms from the El Tatio geothermal field. Achnanthidium exiguum (a), Cocconeis placentula var. euglypta (b), Cocconeis placentula var. lineata (c), Eolimna minima (d), Fragilaria capucina (e), Fragilaria capucina var. vaucheriae (f), Gomphonema angustum (g), Gomphonema parvulum (h). Luticola mollis (i). Navicula gregaria (j), Navicula lauca (k), Navicula parinacota ((l), (m)). 


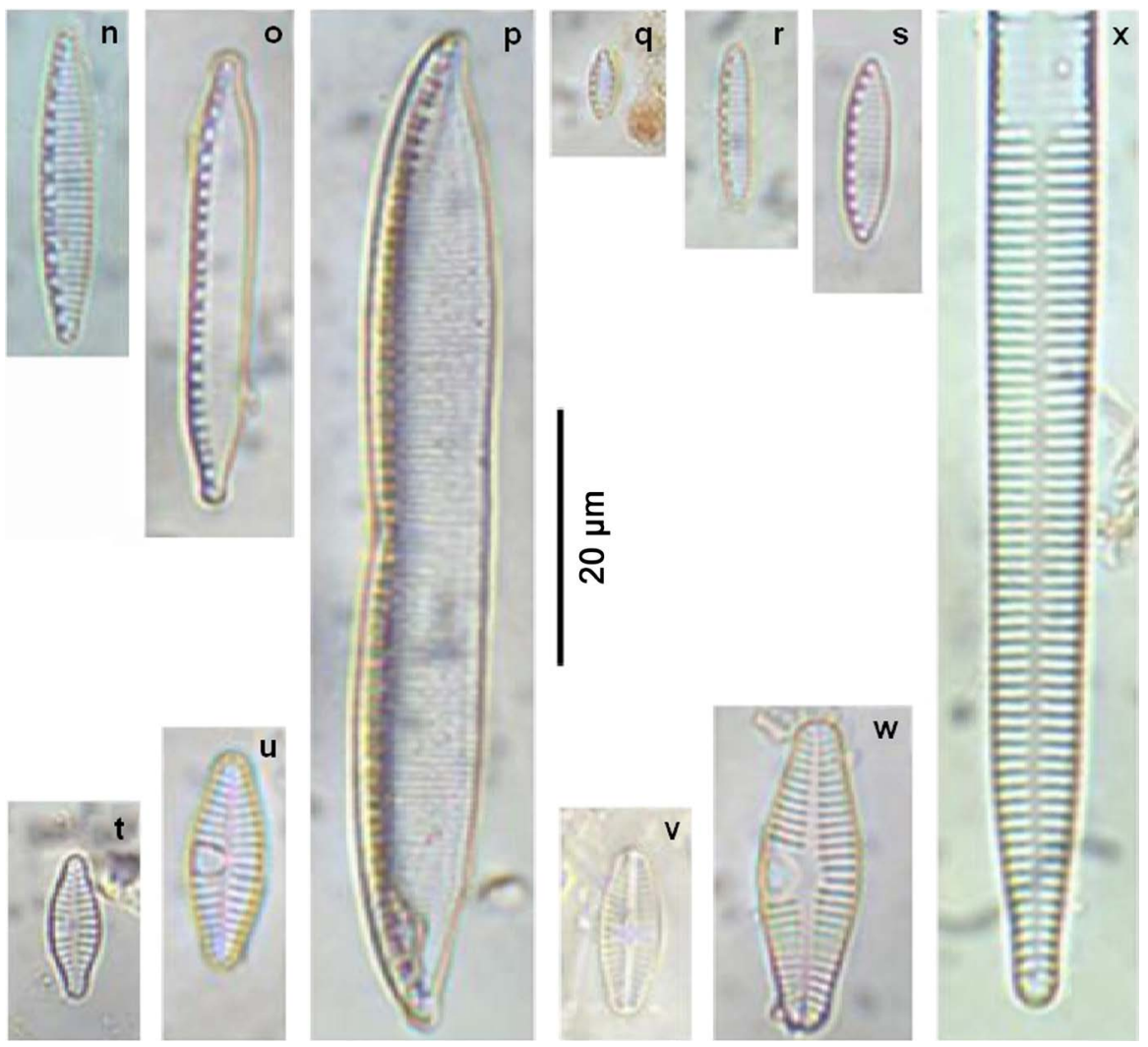

Figure 8. Light microscopy photographs of diatoms from the El Tatio geothermal field. Nitzschia amphibia (n), Nitzschia halloyii (o), Nitzschia hungarica (p), Nitzschia inconspicua ((q), (r)), Nitzschia perminuta (s), Planothidium delicatulum (t), Planothidium frecuentissimum $(\mathrm{u})$, Planothidium lanceolatum $(\mathrm{v}),(\mathrm{w}))$, Ulnaria ulna $(\mathrm{x})$.

Achnanthidium minutissimum (Kützing) Czarnecki, Staurosira subsalina (Hustedt) Lange-Bertalot, Staurosira brevistriata (Grunow) Grunow, Gomphonema pseudoaugur Lange-Bertalot, Navicula salinicola Hustedt, Navicula cincta (Ehrenberg) Ralfs, Navicula tripunctata (Müller) Bory, Nitzschia amphibia (Figure 8(n)), Caloneis bacillum (Grunow) Cleve, and Mayamaea atomus (Kützing) Lange-Bertalot. These taxa were mostly observed in water flow across the north discharge area (e.g. fumaroles and saline systems), and through stream channels until it arrives at the Salado River.

Group III included: Nitzschia fonticola (Grunow) Grunow, Nitzschia palea, Cocconeis placentula var. lineata (Ehrenberg) Van Heurck (Figure 7(c)), Eolimna minima (Figure $7(\mathrm{~d})$ ), Fragilaria capucina var. vaucheriae (Kützing) Lange-Bertalot (Figure 7(f)), Nitzschia inconspicua (Figure 8(q) \& Figure 8(r)), Planothidium lanceolatum (Figure 8(v) \& Figure 8(w)), Planothidium frecuentissimum (Lange-Bertalot) Lange-Bertalot (Figure 8(u)) and Ulnaria ulna (Figure $8(\mathrm{x})$ ). These diatoms were not exclusive at south area, however a high number of taxa were clearly associated to freshwater systems, rivers and the margins of swamp stations. They were dominant in the sparse flora that occurs on both discharge areas (north and south), and formed part of the common flora in freshwater systems at El Tatio geothermal field. 


\subsection{Statistical Model}

Monte Carlo test (999 permutations, $\mathrm{p}<0.05$ ) showed that physical and chemical variables were important diatom predictor (Figure 9). The best species response to significant environmental variables were observed in 39 taxa including all the samples in the CCA analysis. Permutation under the reduced model indicated conductivity, dissolved oxygen and composition of major ions (TP, $\mathrm{HCO}_{3}$, $\mathrm{TKN}$ and $\mathrm{Mg}$ ) as the most important variables with relation to diatom species assemblages ( $p<0.05,999$ permutations). Some taxa were associated to saline systems, the most common were: Fragilaria fasciculata (C.Agardh) Lange-Bertalot, Surirella chilensis, Navicula salinicola, Navicula pseudogracilis Hustedt, Navicula microdigitoradiata Lange-Bertalot, Nitzschia liebetruthii Rabenhorst, Navicymbula pusilla (Grunow) Krammer, Pseudostaurosira brevistriata. These species were correlated with high conductivity and temperature, and concentration of nitrogen and phosphorous. The second group was associated to swamp stations separated from the other freshwater systems. This group included: Navicula cincta, Planothidium frecuentissimum, Mayamaea atomus, Fragilaria capucina var. vaucheriae, Planothidium delicatulum, Lemnicola hungarica (Grunow) Round and Basson, and Halamphora veneta (Kützing) Levkov. Other species associated to this group was Nitzschia perminuta, and reported the highest statistical weight, this species was only observed in swamps and was highly correlated with $\mathrm{SO}_{4}^{2-}$ and $\mathrm{CaCO}_{3}^{-}$water types. The third assemblage was associated

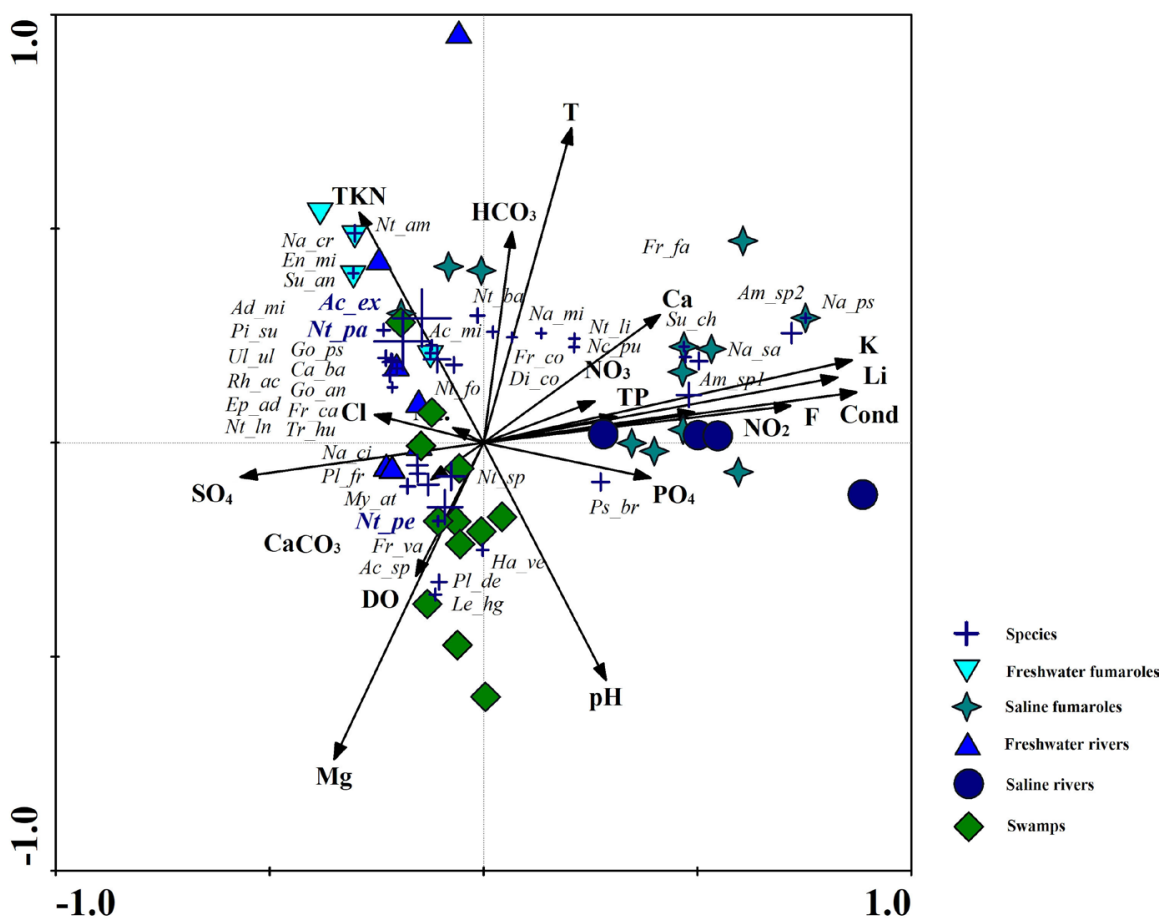

Figure 9. CCA ordination plot of diatom assemblages (using only species that explain 0.1 to 0.33 of the total variance) against selected environmental variables, including weighted average for each species plot (showing in blue bold the species that represent the higher values, species acronyms are described in Table 3 ). 
to freshwater fumaroles and freshwater rivers. This group was more diverse and include: Nitzschia amphibia, Navicula cryptocephala Kützing, Encyonopsis microcephala (Grunow) Krammer, Surirella angusta Kützing, Adlafia minuscula (Grunow) Lange-Bertalot, Pinnularia subrostrata (A. Cleve) Cleve-Euler, Ulnaria ulna, Rhopalodia acuminata Krammer, Epithemia adnata (Kützing) Brébisson, Nitzschia linearis W. Smith, Tryblionella hungarica, Fragilaria capucina Desmazières (Figure 7(e)), Gomphonema angustum C.Agardh (Figure 7(g)), Caloneis bacillum, and Gomphonema pseudoaugur. Other two species were also associated to these systems, Achnanthidium exiguum and Nitzschia palea (Figure 9), and reported the highest statistical weight highly correlated with nitrogen contained in organic substances plus the nitrogen in inorganic ammonia and ammonium $\left(\mathrm{NH}_{3} / \mathrm{NH}_{4}^{+}\right)$.

\subsection{Species Indicator Values}

Multiple sensitive species for significant variables $(\mathrm{p}<0.05)$ were determined using Generalized Additive Model (GAM) and Poisson distribution. Using maximum values and stepwise selection with Akaike Index Criterion (AIC), only 30 species pass the restrictions (the rest of the species were no candidate for additive model because had AIC values lower than null model) and where used to species curve fitted (Table 4). The optima and tolerance was estimate using restrictions (species with statistical weight among 0.01 and 0.33 of the total variance); they were explained by significant variables $(\mathrm{p}<0.05)$. Conductivity optima for those diatoms ranged from 960 to $15,310 \mu \mathrm{S} \mathrm{cm} \mathrm{cm}^{-1}$. Mostly, diatoms that exhibited the highest affinity to $\mathrm{Ca}\left(\mathrm{HCO}_{3}\right)_{2}$ rich waters had high to moderate conductivity optima. In this aspect, the most common taxa were Halamphora acutiuscula, Halamphora atacamae Hustedt, Diadesmis confervacea, Fragilaria fasciculata, Nitzschia lateens Hustedt, Nitzschia palea, Staurosira brevistriata, and Cocconeis placentula var. lineata. Other species indicator values for the most significant variables were described in Table 4.

\section{Discussion}

High seasonal variation both in thermal and chemical properties have been described, and those variables tend to alter diatom assemblages, alternately favoring one species than another. Seasonal rains keep the diatom assemblages in an early successional state, composed of fluctuating 'opportunistic' species (e.g. rare or occasional, Table 3). Initial colonization usually produces a variety of species, none in great abundance but in high diversity [5]-[21]. Gradually, with longer exposure to the strongest dryness a few species are capable of rapid growth, gaining dominance and stability. For example, $A$. exiguum and $S$. pinnata were the most abundant species found in fumaroles and saline rivers, with maximal abundance reported during the dry season. In contrast, $N$. palea had maximal abundance at summer during the seasonal rains. The maximal values for the dominant species in swamps, C. placentula var. euglypta, $N$. gregaria, $N$. inconspicua, $N$. perminuta, and $P$. lanceolatum, were after seasonal rains. 
Table 4. Optima and tolerance values for common thermal/non-thermal associated diatoms, and peak maximum $\left(\mathrm{Cells} / \mathrm{mm}^{3}\right)$ based on a weighted averaging model.

\begin{tabular}{|c|c|c|c|c|c|c|c|c|c|c|c|c|}
\hline Species & $\begin{array}{l}\text { Cond. } \\
\text { Opt. }\end{array}$ & $\begin{array}{l}\text { Cond. } \\
\text { Tol. }\end{array}$ & $\begin{array}{l}\text { Peak } \\
\text { max. }\end{array}$ & $\begin{array}{l}\text { D.O. } \\
\text { Opt. }\end{array}$ & D.O. Tol. & $\begin{array}{l}\text { Peak } \\
\text { max. }\end{array}$ & pH Opt. & pH Tol. & $\begin{array}{l}\text { Peak } \\
\text { max. }\end{array}$ & $\begin{array}{l}\text { T.P. } \\
\text { Opt. }\end{array}$ & T.P. Tol. & $\begin{array}{l}\text { Peak } \\
\text { max. }\end{array}$ \\
\hline $\begin{array}{c}\text { Achnanthes thermalis var. } \\
\text { rumrichorum }\end{array}$ & $7.1 \pm 0.8$ & $3.8 \pm 0.8$ & 35.9 & $4.3 \pm 2.7$ & $0.7 \pm 0.1$ & 13.4 & $5.2 \pm 0.9$ & $1.5 \pm 0.3$ & 4.3 & $0.4 \pm 0.1$ & $0.1 \pm 0.1$ & 15.9 \\
\hline Achnanthidium exiguum & $8.5 \pm 2.8$ & $3.6 \pm 0.6$ & 110.4 & $6.8 \pm 0.5$ & $2.7 \pm 1.2$ & 120.5 & $5.8 \pm 0.7$ & $1.8 \pm 0.5$ & 103.7 & $0.5 \pm 0.2$ & $0.6 \pm 0.2$ & 152.8 \\
\hline $\begin{array}{l}\text { Achnanthidium } \\
\text { minutissimum }\end{array}$ & $5.2 \pm 0.1$ & $0.9 \pm 0.3$ & 20.1 & $5.6 \pm 0.2$ & $2.9 \pm 0.2$ & 18.2 & $5.3 \pm 0.5$ & $1.9 \pm 0.5$ & 58.5 & $0.2 \pm 0.1$ & $0.1 \pm 0.1$ & 18.2 \\
\hline Adlafia minuscula & $2.1 \pm 0.2$ & $2.2 \pm 0.2$ & 11.5 & $8.4 \pm 0.5$ & $2.7 \pm 0.5$ & 28.7 & $5.1 \pm 0.6$ & $0.7 \pm 0.2$ & 18.4 & $0.9 \pm 0.4$ & $0.1 \pm 0.1$ & 20.7 \\
\hline Amphora pediculus & $1.1 \pm 0.1$ & $0.4 \pm 0.2$ & 114.1 & $6.8 \pm 0.2$ & $0.4 \pm 0.2$ & 49.7 & $7.7 \pm 1.1$ & $0.9 \pm 0.1$ & 51.9 & $0.6 \pm 0.3$ & $0.2 \pm 0.1$ & 15.9 \\
\hline $\begin{array}{c}\text { Cocconeis placentula var. } \\
\text { euglypta }\end{array}$ & $7.1 \pm 2.5$ & $3.5 \pm 0.8$ & 49.7 & $7.8 \pm 0.2$ & $2.9 \pm 0.1$ & 65.9 & $7.3 \pm 1.8$ & $2.3 \pm 0.8$ & 10.8 & $0.6 \pm 0.1$ & $0.7 \pm 0.1$ & 22.8 \\
\hline Diadesmis confervacea & $11.9 \pm 0.6$ & $4.7 \pm 1.1$ & 22.7 & $5.9 \pm 0.8$ & $0.6 \pm 0.1$ & 23.8 & $7.9 \pm 1.9$ & $0.5 \pm 0.1$ & 15.5 & $0.3 \pm 0.1$ & $0.2 \pm 0.1$ & 22.9 \\
\hline Fragilaria capucina & $3.4 \pm 1.2$ & $5.8 \pm 4.4$ & 15.5 & $11.5 \pm 0.1$ & $2.4 \pm 0.2$ & 46.5 & $7.3 \pm 1.7$ & $0.8 \pm 0.4$ & 20.2 & $0.8 \pm 0.3$ & $0.2 \pm 0.1$ & 11.8 \\
\hline $\begin{array}{c}\text { Fragilaria capucina var. } \\
\text { vaucheriae }\end{array}$ & $2.3 \pm 0.1$ & $2.8 \pm 0.9$ & 51.6 & $8.8 \pm 0.4$ & $2.3 \pm 0.6$ & 34.8 & $7.8 \pm 1.2$ & $1.2 \pm 0.3$ & 15.0 & $0.2 \pm 0.1$ & $0.1 \pm 0.1$ & 150.1 \\
\hline Fragilaria fasciculata & $9.2 \pm 0.2$ & $3.8 \pm 0.2$ & 23.2 & $4.7 \pm 0.2$ & $0.7 \pm 0.3$ & 16.2 & $7.5 \pm 1.1$ & $1.6 \pm 0.5$ & 18.2 & $0.2 \pm 0.1$ & $0.4 \pm 0.1$ & 6.5 \\
\hline Halamphora acutiuscula & $13.5 \pm 0.8$ & $3.5 \pm 0.1$ & 130.9 & $5.4 \pm 0.6$ & $1.5 \pm 0.4$ & 45.2 & $9.9 \pm 2.7$ & $2.4 \pm 0.7$ & 598.3 & $0.2 \pm 0.1$ & $0.3 \pm 0.1$ & 18.3 \\
\hline Halamphora atacamae & $13.2 \pm 0.9$ & $5.1 \pm 1.6$ & 32.2 & $5.6 \pm 0.9$ & $1.6 \pm 0.4$ & 11.2 & $7.7 \pm 1.5$ & $0.8 \pm 0.2$ & 11.7 & $0.2 \pm 0.1$ & $0.2 \pm 0.1$ & 7.1 \\
\hline Halamphora veneta & $11.2 \pm 0.8$ & $4.5 \pm 1.3$ & 20.5 & $7.5 \pm 0.2$ & $2.8 \pm 0.3$ & 28.7 & $7.3 \pm 1.8$ & $0.8 \pm 0.1$ & 11.5 & $0.2 \pm 0.1$ & $0.1 \pm 0.1$ & 11.8 \\
\hline Lemnicola hungarica & $3.3 \pm 0.1$ & $1.6 \pm 0.1$ & 17.2 & $6.5 \pm 0.1$ & $0.8 \pm 0.1$ & 13.1 & $7.7 \pm 1.3$ & $0.7 \pm 0.4$ & 10.5 & $0.1 \pm 0.1$ & $0.1 \pm 0.1$ & 14.7 \\
\hline Mayamaea atomusm & $2.3 \pm 0.5$ & $1.3 \pm 0.3$ & 51.2 & $4.9 \pm 0.2$ & $0.5 \pm 0.1$ & 23.2 & $5.5 \pm 1.1$ & $1.7 \pm 0.5$ & 42.5 & $0.3 \pm 0.1$ & $0.3 \pm 0.1$ & 34.4 \\
\hline Navicula cincta & $2.4 \pm 0.2$ & $1.4 \pm 0.2$ & 36.4 & $4.4 \pm 0.7$ & $1.2 \pm 0.1$ & 46.5 & $5.8 \pm 1.2$ & $1.9 \pm 0.6$ & 293.3 & $0.1 \pm 0.1$ & $0.2 \pm 0.1$ & 45.3 \\
\hline Navicula gregaria & $1.8 \pm 0.4$ & $0.3 \pm 0.1$ & 270.2 & $6.8 \pm 0.5$ & $1.6 \pm 0.3$ & 75.2 & $8.5 \pm 2.6$ & $1.7 \pm 0.5$ & 293.3 & $0.4 \pm 0.1$ & $0.2 \pm 0.1$ & 353.6 \\
\hline Navicula tripunctata & $1.7 \pm 0.3$ & $0.2 \pm 0.1$ & 224.4 & $6.8 \pm 0.2$ & $0.7 \pm 0.3$ & 53.1 & $7.8 \pm 2.7$ & $2.5 \pm 1.2$ & 183.8 & $0.3 \pm 0.1$ & $0.2 \pm 0.1$ & 58.2 \\
\hline Nitzschia amphibia & $8.1 \pm 0.5$ & $3.9 \pm 1.5$ & 192.3 & $5.7 \pm 0.1$ & $1.8 \pm 0.2$ & 2242.6 & $4.7 \pm 1.3$ & $0.8 \pm 0.3$ & 3440.3 & $1.8 \pm 0.3$ & $0.8 \pm 0.5$ & 935.2 \\
\hline Nitzschia fonticola & $0.8 \pm 0.3$ & $0.6 \pm 0.1$ & 1183.8 & $8.2 \pm 0.5$ & $1.6 \pm 0.3$ & 153.4 & $4.6 \pm 0.8$ & $1.4 \pm 0.5$ & 182.2 & $0.7 \pm 0.1$ & $0.8 \pm 0.2$ & 565.5 \\
\hline Nitzschia lateens & $15.3 \pm 0.9$ & $5.2 \pm 1.3$ & 26.5 & $5.7 \pm 0.3$ & $0.9 \pm 0.1$ & 22.4 & $6.8 \pm 1.5$ & $0.7 \pm 0.2$ & 92.5 & $0.5 \pm 0.1$ & $0.6 \pm 0.1$ & 12.1 \\
\hline Nitzschia palea & $14.7 \pm 2.4$ & $4.9 \pm 3.1$ & 276.7 & $5.4 \pm 0.2$ & $1.8 \pm 0.4$ & 212.6 & $4.8 \pm 0.7$ & $1.9 \pm 0.9$ & 87.5 & $1.8 \pm 0.5$ & $0.9 \pm 0.4$ & 135.2 \\
\hline Nitzschia perminuta & $2.8 \pm 0.1$ & $1.8 \pm 0.2$ & 469.5 & $7.6 \pm 0.5$ & $1.4 \pm 0.6$ & 92.1 & $6.8 \pm 1.2$ & $1.8 \pm 0.5$ & 98.8 & $0.3 \pm 0.1$ & $0.6 \pm 0.1$ & 150.5 \\
\hline Nitzschia pusilla & $11.8 \pm 0.2$ & $4.8 \pm 1.1$ & 55.2 & $5.4 \pm 0.5$ & $1.5 \pm 0.3$ & 102.6 & $5.8 \pm 1.2$ & $0.5 \pm 0.2$ & 14.4 & $0.7 \pm 0.4$ & $0.6 \pm 0.2$ & 12.8 \\
\hline Planothidium delicatulum & $2.1 \pm 0.3$ & $0.9 \pm 0.2$ & 60.7 & $7.8 \pm 0.3$ & $0.8 \pm 0.4$ & 54.2 & $7.2 \pm 1.6$ & $1.8 \pm 0.5$ & 611.7 & $2.1 \pm 0.5$ & $1.1 \pm 0.5$ & 116.4 \\
\hline Planothidium lanceolatum & $2.7 \pm 0.8$ & $0.8 \pm 0.1$ & 1093.3 & $7.7 \pm 0.2$ & $1.1 \pm 0.2$ & 175.0 & $5.9 \pm 1.5$ & $1.3 \pm 0.5$ & 120.2 & $1.2 \pm 0.5$ & $0.7 \pm 0.3$ & 105.2 \\
\hline Rhoicosphenia abbreviata & $2.9 \pm 0.4$ & $0.8 \pm 0.1$ & 32.7 & $7.5 \pm 0.4$ & $0.8 \pm 0.3$ & 13.4 & $7.9 \pm 1.2$ & $0.8 \pm 0.1$ & 24.8 & $1.6 \pm 0.5$ & $0.9 \pm 0.5$ & 28.6 \\
\hline Staurosira brevistriata & $6.5 \pm 0.8$ & $2.9 \pm 0.1$ & 53.4 & $4.1 \pm 0.5$ & $1.7 \pm 0.5$ & 35.1 & $7.8 \pm 1.5$ & $1.8 \pm 0.5$ & 28.5 & $1.8 \pm 0.5$ & $0.7 \pm 0.2$ & 33.2 \\
\hline Ulnaria ulna & $3.6 \pm 0.2$ & $1.1 \pm 0.5$ & 17.6 & $8.6 \pm 0.9$ & $2.2 \pm 0.4$ & 33.7 & $5.6 \pm 1.1$ & $2.5 \pm 0.7$ & 24.4 & $0.9 \pm 0.1$ & $0.4 \pm 0.1$ & 18.5 \\
\hline
\end{tabular}

Dissolved Oxygen (DO) and Total Phosphorous (TP) values in (mg/L); Conductivity $(\mathrm{mS} / \mathrm{cm})$. 
The epiphytic Cocconeis placentula (and varieties) is considered a euryhaline and tychonoplanktonic species [22]. In Bolivia, it has been recorded in saline systems (44- $80 \mathrm{~g} \mathrm{l}^{-1}$ ), and diatom stratigraphy works by [22] and [23]. It now occurs in a large range of salinity, in large permanent freshwater water bodies colonized by macrophytes (essentially Isoetes) and also reported in the glacial valley of Hichu Kkota in a shallow salt lake (e.g. $15 \mathrm{~g} \mathrm{l}^{-1}$ salinity), and in the Lake Poopó (40 $\mathrm{g} \mathrm{l}^{-1}$ salinity). In Chile, this species have been described in a large range of salinity, from sediments and littoral habitat of the Chungará Lake [24], to variable saline systems in the Salar de Atacama [25]. C. placentula var. euglypta (Figure $7(\mathrm{~b})$ ) and C. placentula var. lineata (Ehrenberg) Van Heurck (Figure $7(\mathrm{c})$ ) are widespread in a variety of environments, from clean to enriched streams [6], described commonly as epiphytic (Patrick and Reimer 1966), but also appear to have an important ecological function in a variety of aquatic environments, especially in littoral water bodies colonized by macrophytes. N. cincta (Ehrenberg) Ralfs, an aerophilous species according to [26], lives at around $10 \mathrm{~g} \mathrm{l}^{-1}$ salinity in Bolivia. This species was more associated with fumaroles and saline rivers $\left(>10^{3}\right.$ cells $\left./ \mathrm{mm}^{3}\right)$ than freshwater systems studied.

Some studies have focused on assessing the impact of environmental and spatial drivers in explaining the differences in diatom species richness and composition patterns. However, these aspects in microbial communities are poorly know especially in extreme environments. In New Zealand, according to [7] 144 taxa in thermal and mixed thermal/non-thermal waters were dominated by the genera Nitzschia (24), Pinnularia (16), Navicula (10), and Surirella (7). In this work, we reported 102 taxa in thermal and mixed thermal/non-thermal waters dominated by the genera Nitzschia (16), Navicula (13), Gomphonema (7), Planothidium (6), and Denticula (5). In Iceland 178 taxa were identified in hot springs, where Diploneis elliptica (Kützing) Cleve, N. amphibia, Pinnularia borealis Ehrenberg, and E. adnata occurred in 50\% of the samples [27]. However, other authors suggested that nine species were found only in hot springs in Iceland [28]. In contrast, other report [29] argued that exclusive geothermal diatoms do not exist and pointed out that other studies [27] [30] had detected these taxa in other habitats as well. In this context, we found only 18 taxa exclusive of warm-fumarole springs.

Many of the taxa reported in the El Tatio geothermal field were described by many authors in a wide range of thermal/non-thermal environments. For example, $A$. exiguum has a worldwide distribution in hot springs [31]. S. pinnata predominated in shallow arctic lakes and is more competitive in lakes with long periods of ice cover [32] [33]. P. lanceolatum is common and widespread in the hot springs of New Zealand and can be found in a variety of water chemistries [6]. It typically prefers oxygen-rich waters of neutral to slightly alkaline $\mathrm{pH}$ [13]. This condition was similar to this report, especially in freshwater systems. In this case it was very restrictive to pore-water electrolytes. $N$. palea is common in many environments worldwide and described as a sensitive taxon in New Zealand [6] and variable environments in Alaska [34]. We reported the maximal 
abundance for this taxa in fumaroles and saline rivers (Table 3). The distribution of $H$. atacamae, $H$. coffeaeformis (C. Agardh) Levkov, Denticula elegans Kützing, Denticula thermalis Kützing, and $S$. chilensis were coincident with the photosynthetic microbial communities described in a near local high-altitude saline basin [35].

According to [22] species of the genus Fragilaria were dominant in the freshwater environments of the Hichu Kkota glacial valley in Bolivia. It is rare in aerophilic habitats, but particularly abundant (always more than 50\%) in flowing streams (50 - $100 \mathrm{~cm}$ deep) which are scattered in the Juncacae peat bogs (bofedales). Fragilaria are here assigned as tychoplanktonic; some species are considered as benthic in lakes and ponds [11]. They prefer low conductivity in lotic environments [32]. However, we described four taxa, F. capucina (Figure 7(e)), F. capucina var. vaucheriae (Figure 7(f)), Fragilaria construens, and F. fasciculata above $10,000 \mu \mathrm{S} \mathrm{cm}^{-1}$ conductivity in some rivers and swamps from the El Tatio geothermal field. In Bolivia, F. fasciculata and F. capucina were observed in mixed saline and freshwaters. F. fasciculata was also described in the Salar of Uyuni (0.9 - $30 \mathrm{~g} \mathrm{l}^{-1}$ salinity), and Lake Poopó (14 - $30 \mathrm{~g} \mathrm{l}^{-1}$ salinity, $50-200 \mathrm{~cm}$ water depth). Moreover, F. capucina and F. construens was reported in the streams of the Hichu Kkota valley in Bolivia.

The fossil benthic species composition in core sediment from Lake Titicaca [23], indicated abundant taxa in common with the El Tatio such as C. placentula, $N$. veneta, Navicula lauca Rumrich and Lange-Bertalot (Figure $7(\mathrm{k})$ ), P. lanceolatum (Figure 8(v) \& Figure 8(w)) and S. pinnata. High percentage values of fossil periphytic diatoms, mainly epiphytes, were described in the Quillagua formation (Atacama Desert); indicating permanent palustrine conditions with abundance of macrophytes and very shallow mesohaline water bodies [36]. The most relevant species including saline forms were similar to the species described in the El Tatio, Achnanthes atacamae Hustedt, Brachysira aponina Kützing, Cocconeis placentula Ehrenberg, Denticula valida (Pedicino) Grunow, $N$. amphibia, $N$. inconspicua, $N$. valdecostata, and Denticula kuetzingii Grunow. The freshwater species described in common with the El Tatio were $U$. ulna and $N$. palea, which tolerate great variation in oxygen and moderate to low electrolyte concentration [17] [18] [19]. Another freshwater species described was Achnanthes lanceolata Smith, and two peaks of F. brevistriata and S. subsalina, interpreted as indicators of freshwater inputs. Moreover, a peak in Achnanthes thermalis (Rabenhorst) Schönfeldt was indicated as the influence of water discharges from nearby hot springs [13] [36]. The presence of Cymbella pusilla Grunow, described as an indicator of $\mathrm{CaSO}_{4}$-rich water type [8], suggests a similar condition with the actual diatom flora described in the El Tatio geothermal field.

Recently, interesting patterns related with diatom size fractions has been evaluated to interpret their relations to environmental variables in a global scale [37]. The maximum growth factor can vary by a factor of two, even within a given species of diatom, increasing with size as cells are transformed from pre to 
post-auxospore cells. Similarly, when mean size cell decreases in diatom because of the reduction of the frustule size over succeeding generations, growth rates decrease [38]. These aspects can influence the diatom size-fraction in hostile environments. As expected, the 20 to $200 \mu \mathrm{m}$-size fraction in El Tatio contained the highest numbers of diatom taxa (53 species) although an unexpectedly high number (47 species) were also found in the smaller size fraction, belonging to smaller species (e.g., Achnanthidium, Eolimna, and Planothidium). The 5 to 20 $\mu \mathrm{m}$-size fraction was an important component more associated to brackish (except for stations E1-E3 during winter season) and saline systems (Figure 10). Apparently, the aquatic systems in El tatio geothermal field are highly restrictive for larger size-fraction $180-2000 \mu \mathrm{m}$, only two species, including rosette-forming diatom $U$. ulna and the unicellular species $S$. chilensis, were found exclusively in freshwater systems.

As indicated by various studies, some habitats are highly stressful and so few

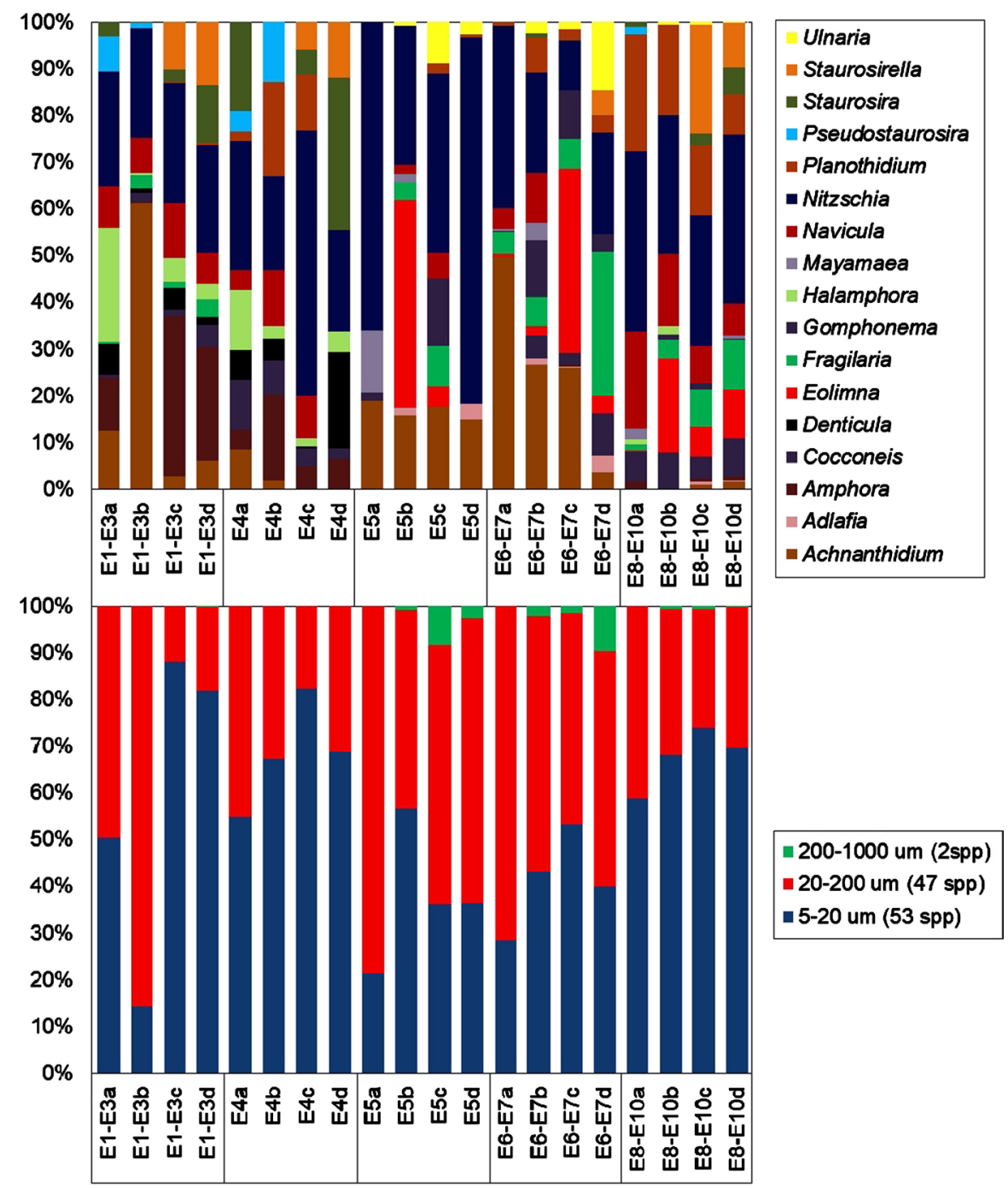

Figure 10. Seasonal change of the most representative genera ( $>1 \%$ total abundance per sample) and size fractions variability. 
microorganisms are adapted for life there, this result in many of them are unique or rare [35] [39] [40]. Water bodies in different geographic regions often show considerable variability with regard to their diatom richness/composition patterns. These patterns would response to altitude and latitudinal variations (solar radiation, cloudiness, temperature, desiccation and seasonality) superimposed on local habitat controls (current velocity, water chemistry, and substrate types). In many studies, cosmopolitan diatom taxa are described, suggesting easy dispersal mechanisms [37]. However, regional studies of geothermal diatoms have shown taxonomic variability between thermal/non-thermal waters at varied local to regional scales [6]. Our study clearly shown exclusive diatom taxa related to thermal waters, however, salinity and other local environmental condition appear to have a major influence in the structure of diatom assemblages. In contrast, cosmopolitan taxa are widespread, but only some occurs in specialized settings such as the hot springs.

\section{Conclusion}

Diatom floras in the El Tatio geothermal field vary in terms of beta diversity and abundance, and several taxa were found in all the systems studied. Nevertheless, the study areas could still be distinguished by: 1) the dominance of $A$. exiguum, A. minutissimum, D. subtilis, D. thermalis, H. acutiuscula, N. salinicola, N. amphibia and $N$. bacillum in fumaroles, with the common occurrence of teratological forms; 2) the common occurrence of $A$. exiguum, $C$. placentula var. lineata and $N$. palea in rivers; 3 ) the common occurrence of $C$. placentula var. euglypta, $N$. gregaria, $N$. tripunctata, $N$. inconspicua and $P$. lanceolatum in swamps. These variations probably reflect the combined influence of heterogeneity and differences in temperature, water chemistry brought about by contrasts in lithologies, and flow paths of water courses. Stations were distinguished from saline to freshwater systems; cluster analyses indicate three distinct floral groups, and CCAs indicate that the diatoms can be related to a variety of environmental variables. Inference models show strong correlations between diatoms and conductivity, TP (total phosphorous), $\mathrm{NO}_{3}^{-}, \mathrm{HCO}_{3}^{-}, \mathrm{Mg}^{2+}$ and $\mathrm{DO}$ (dissolved oxygen). Other factors such as substrate type and current velocity are probably important factors in the local community structures. The diatoms were well preserved in the El Tatio and would potentially provide valuable information on groundwater chemistry in studies of similar spring systems. However, their use in such investigations would also depend on the important preservation of these unique environments.

\section{Acknowledgements}

This work was supported by Fondecyt project 1140543, institutional research project Universidad de Chile. The authors would like to express their sincere thanks to the members of the limnology laboratory for assistance and critical comments. 


\section{References}

[1] Fernández-Turiel, J.L., García-Valles, M., Gimeno-Torrente, D., Saavedra-Alonso, J. and Martinez-Manent, S. (2005) The Hot Spring and Geyser Sinters of El Tatio, Northern Chile. Sedimentary Geology, 180, 125-147. https://doi.org/10.1016/j.sedgeo.2005.07.005

[2] Glenon, J.A. and Paff, R.M. (2003) The Extraordinary Thermal Activity of El Tatio Geyser Field, Antofagasta Region, Chile. The GOSA Transactions, 8, 31-78.

[3] Romero, L., Alonso, H., Campano, P., Fanfani, L., Cidu, R., Dadea, C., et al. (2003) Arsenic Enrichment in Waters and Sediments of the Rio Loa (Second Region, Chile). Applied Geochemistry, 18, 1399-1416. https://doi.org/10.1016/S0883-2927(03)00059-3

[4] Cusicanqui, H., Mahon, W.A.J. and Ellis, A.J. (1976) The Geochemistry of the El Tatio Geothermal Field, Northern Chile. 2nd United Nations Geothermal Symposium Proceedings, Berkeley.

[5] Weed, W.H. (1889) The Diatom Marshes and Diatom Beds of the Yellowstone National Park. Botanical Gazette, 14, 117-120. https://doi.org/10.1086/326403

[6] Owen, R.B., Renaut, R.W. and Jones, B. (2008) Geothermal Diatoms: A Comparative Study of Floras in Hot Springs Systems of Iceland, New Zealand, and Kenya. Hydrobiologia, 610, 175-192. https://doi.org/10.1007/s10750-008-9432-y

[7] Cassie, V. and Cooper, R.C. (1989) Algae of New Zealand Thermal Areas. J. Cramer, Berlin, 78, 1-261.

[8] Gasse, F. (1986) East African Diatoms. Taxonomy, Ecological Distribution. Bibliotheca Diatomologica. J. Crammer, Berlin.

[9] Cocquyt, C. (1999) Seasonal Variations of Epilithic Diatom Communities in the Northern Basin of Lake Tanganyika. Systematics and Geography of Plants, 69, 265-273. https://doi.org/10.2307/3668547

[10] Mpawenayo, B. and Mathooko, J.M. (2005) The Structure of Diatom Assemblages Associated with Cladophora and Sediments in a Highland Stream in Kenya. Hydrobiologia, 544, 55-67. https://doi.org/10.1007/s10750-004-8333-y

[11] Rumrich, U., Lange-Bertalot, H. and Rumrich, M. (2000) Diatomeen der Anden (Von Venezuela bis Patagonien/Tierra del Fuego und zwei weitere Beitráge). Iconographia Diatomologica.

[12] Van de VijVer, B. and Cocquyt, C. (2009) Four New Diatom Species from La Calera Hot Spring in the Peruvian Andes (Colca Canyon). Diatom Research, 24, 209-223. https://doi.org/10.1080/0269249X.2009.9705792

[13] Patrick, R. and Reimer, C.W. (1975) Entomoneidaceae, Cymbellaceae, Gomphonemaceae, Epithemiaceae. In: Monographs of the Academy of Natural Sciences of Philadelphia, Ed., The Diatoms of the United States, Vol. II/1, Philadelphia.

[14] Battarbee, R.W., Jones, V.J., Flower, R.J., Cameron, N.G., Bennion, H., Carvalho, L. and Juggins, S. (2001) Diatoms. In: Smol, J.P., Birks, H.J.B. and Last, W.M., Eds., Terrestrial, Algal and Siliceous Indicators, Kluwer Academic Publishers, Dordrecht.

[15] Gros, N. (2003) Ion Chromatographic Analyses of Sea Waters, Brines and Related Samples. Water, 5, 659-676. https://doi.org/10.3390/w5020659

[16] APHA (2005) Standard Methods for the Examination of Water and Wastewater. American Public Health Association, Washington DC.

[17] Krammer, K. and Lange-Bertalot, H. (1986) Bacillariophyceae 1. Teil: Naviculaceae. In: Ettl, H., Gerloff, J., Heynig, H. and Mollenhauer, D., Eds., Süsswasserflora von 
Mitteleuropa, Vol. 2/1, G. Fischer, Stuttgart and New York.

[18] Krammer, K. and Lange-Bertalot, H. (1988) Bacillariophyceae. 2. Teil: Bacillariaceae, Epithemiaceae, Surirellaceae. In: Ettl, H., Gerloff, J., Heynig, H. and Mollenhauer, D., Eds., Süsswasserflora von Mitteleuropa, Vol. 2/2, VEB Gustav Fischer Verlag, Jena.

[19] Krammer, K. and Lange-Bertalot, H. (1991) Bacillariophyceae. 4. Teil: Achnanthaceae, Kritische Ergänzungen zu Navicula (Lineolatae) und Gomphonema, Gesamtliteraturverzeichnis Teil 1-4. In: Ettl, H., Gerloff, J., Heynig, H. and Mollenhauer, D., Eds., Süsswasserflora von Mitteleuropa, Vol. 2/4, Gustav Fischer Verlag, Stuttgart, Jena.

[20] Ter Braak, C.J.F. and Smilauer, P. (2002) CANOCO Reference Manual and Canodraw for Windows User's Guide: Software for Canonical Community Ordination. Ithaca, New York.

[21] Williams, D.M. and Kociolek, J.P. (2007) Pursuit of a Natural Classification of Diatoms: History, Monophyly and the Rejection of Paraphyletic Taxa. European Journal of Phycology, 42, 313-319. https://doi.org/10.1080/09670260701419921

[22] Servant-Vildary, S., Servant, M. and Jimenez, O. (2001) Holocene Hydrological and Climatic Changes in the Southern Bolivian Altiplano according to Diatom Assemblages in Paleowetlands. Hydrobiologia, 466, 267-277.

https://doi.org/10.1023/A:1014557417689

[23] Fritz, S.C., Baker, P.A., Seltzer, G.O., Ballantyne, A., Tapia, P.M., Cheng, H. and Edwards, R.L. (2007) Quaternary Glaciations and Hydrologic Variation in the South American Tropics as Reconstructed from the Lake Titicaca Drilling Project. Quaternary Research, 68, 410-420. https://doi.org/10.1016/j.yqres.2007.07.008

[24] Hernández, A., Bao, R., Giralt, S., Leng, M.J., Barker, P.A., Sáez, A., Pueyo, J.J., Moreno, A., Valero-Garcés, B.L. and Sloane, H.J. (2008) The Palaeohydrological Evolution of Lago Chungará (Andean Altiplano, northern Chile) during the Lateglacial and Early Holocene using Oxygen Isotopes in Diatom Silica. Journal of Quaternary Science, 23, 351-363. https://doi.org/10.1002/jqs.1173

[25] Díaz, C.P. and Maidana, N.I. (2005) Diatoms of the Atacama and Punta Negra Salars, II Región-Chile. Centro de Ecología Aplicada, Santiago.

[26] Denys, L. (1994) Diatom Assemblages along a Former Intertidal Gradient a Palaeoecological Study of a Subboreal Clay Layer (Western Coastal Plain. Belgium). Netherland Journal of Aquatic Ecology, 28, 85-96. https://doi.org/10.1007/BF02334249

[27] Østrup, E. (1918) Fresh-Water Diatoms from Iceland. Part I. The Botany of Iceland, II $1(1), 1-98$.

[28] Schwabe, G.H. (1936) Beitrage zur Kenntnis islandischer Thermalbiotope. Archiv fur Hydrobiologie, 6, 151-352.

[29] Petersen, J.B. (1946) Algae Collected by Eric Hulten on the Swedish Kamchatka Expedition, 1920-22, Especially from Hot Springs. Biol. Medd. Dan. Vid. Selsk, 20, 3-122.

[30] Petersen, J.B. (1928) Algefloraen i nogle Jordprver fra Island. Dansk Botanisk Arkiv, 5, 1-23.

[31] Fairchild, E. and Sheridan, R.P. (1974) A Physiological Investigation of the Hot Spring Diatom, Achanates exigua Grünow. Journal of. Phycology, 10, 1-4.

[32] Smol, J.P. (1988) Paleoclimate Proxy Data from Freshwater Arctic Diatoms. Verhandlungen der Internationalen Vereinigung von Limnologen, 23, 837-844. 
https://doi.org/10.1080/03680770.1987.11899722

[33] Rühland, K. and Smol, J.P. (1998) Limnological Characteristics of 70 Lakes Spanning Arctic Treeline from Coronation Gulf to Great Slave Lake in the Central Northwest Territories, Canada. Internationale Revue der gesamten, Hydrobiologie, 83, 183-203. https://doi.org/10.1002/iroh.19980830302

[34] Foged, N. (1981) Diatoms in Alaska. Bibliotheca Phycologica, 53, 1-317.

[35] Angel, A., Vila, I. and Herrera, V. (2016) Extremophiles: Photosynthetic Systems in a High Altitude Saline Basin (Altiplano, Chile). Aquatic International Research, 8, 91-108. https://doi.org/10.1007/s40071-016-0121-6

[36] Bao, R., Sáez, A., Servant-Vildary, S. and Cabrera, L. (1999) Lake-Level and Salinity Reconstruction from Diatom Analyses in Quillagua Formation (Late Neogene, Central Andean Forearc, Northern Chile). Palaeogeography, Palaeoclimatology, Palaeoecology, 153, 309-335. https://doi.org/10.1016/S0031-0182(99)00066-8

[37] Malviya, S., Scalco, E., Audic, S., Vincent, F., Veluchamy, A., Poulain, J., et al. (2016) Insights into Global Diatom Distribution and Diversity in the World's Ocean. Proceeding of the National Academy of Sciences of the United States of America, 113, E1516-E1525. https://doi.org/10.1073/pnas.1509523113

[38] Chisholm, S.W. and Costello, J.C. (1981) Influence of Environmental Factors and Population Composition on the Timing Cell Division in Thalassiosira fluviatilis (Bacillariophyceae) Grown Light/Dark Cycles. Journal of Phycology, 16, 375.

[39] Dorador, C., Vila, I., Witzel, K. and Imhoff, J.F. (2013) Bacterial and Archaeal Diversity in High Altitude Wetlands of the Chilean Altiplano. Fundamental and Applied Limnology, 182, 135-159. https://doi.org/10.1127/1863-9135/2013/0393

[40] Aguilar, P., Acosta, E., Dorador, C. and Sommaruga, R. (2016) Large Differences in Bacterial Community Composition among Three Nearby Extreme Waterbodies of the High Andean Plateau. Frontiers in Microbiology, 7, 976.

https://doi.org/10.3389/fmicb.2016.00976 